\title{
Unitary equivalence of operator-valued multishifts
}

\author{
Rajeev Gupta, Surjit Kumar, and Shailesh Trivedi
}

\begin{abstract}
We systematically study various aspects of operator-valued multishifts. Beginning with basic properties, we show that the class of multishifts on the directed Cartesian product of rooted directed trees is contained in that of operator-valued multishifts. Further, we establish circularity, analyticity and wandering subspace property of these multishifts. In the rest part of the paper, we study the function theoretic behaviour of operator-valued multishifts. We determine the bounded point evaluation, reproducing kernel structure and the unitary equivalence of operator-valued multishifts with invertible operator weights. In contrast with a result of Lubin, it appears that the set of all bounded point evaluations of an operator-valued multishift may be properly contained in the joint point spectrum of the adjoint of underlying multishift.
\end{abstract}

\section{Introduction}

Shift operators constitute a pivotal part of operator theory. The class of shift operators is rich enough to understand various notions in operator theory. There is tremendous literature on these operators $(\mathbf{1 3},[\mathbf{1 4},[\mathbf{1 5},[\mathbf{2 0},[\mathbf{3 2},[\mathbf{1 7},[\mathbf{2 1}$, $\mathbf{3 0}, \mathbf{3}, \mathbf{2 8})$ and equally huge literature exists on their generalized notions ([25, [26, 24, 22, 23, 12, 27, 7], 4, 5], 6). Two significant generalizations of classical weighted shift appeared in $\mathbf{2 4}$ and $\mathbf{2 3}$. The notion of shift operator introduced in 24] is commonly known as classical multishift which extends the notion of classical weighted shift into multivariable settings. On the other hand, the notion of shift operator introduced in $\mathbf{2 3}$ is called weighted shift on directed tree which incorporates discrete structures (directed graphs) to give a broader picture of classical weighted shifts. The recently introduced notion of multishifts on directed Cartesian product of rooted directed trees in [5] unifies both the aforementioned notions in the context of unilateral shifts. Another generalization of classical unilateral weighted shift namely, operator-valued unilateral weighted shift, appeared in 25. Hence one may ask whether there is any notion of shift which unifies all of the aforementioned notions of shift. In this regard, there comes the notion of operator-valued multishifts. It turns out that the class of multishifts on directed Cartesian product of rooted directed trees is contained in that of operator-valued multishifts (see Proposition 2.6). Further, the class of operator-valued multishifts with invertible operator weights turns out to be a rich source of counter-examples to the von Neumann's inequality (see [18). One can not expect such phenomenon in the class of classical multishifts with non-zero weights (see [19]).

2010 Mathematics Subject Classification. Primary 47A13, 47B37, Secondary 46E22, 46E40.

Key words and phrases. operator-valued multishift, circularity, operator-valued reproducing kernel, bounded point evaluation, wandering subspace property.

The work of all the authors was supported by Inspire Faculty Fellowship (Ref. No. DST/INSPIRE/04/2017/002367, DST/INSPIRE/04/2016/001008, DST/INSPIRE/04/2018/000338 respectively). 
In this paper, we systematically study the various aspects of operator-valued multishifts. Starting with prerequisites and the formal definition, we discuss boundedness, commutativity and the moments of operator-valued multishifts. Further, we show that the class of multishifts on directed Cartesian product of rooted directed trees is contained in that of operator-valued multishifts. This constitutes the second section and a partial converse of the aforementioned inclusion concludes this section. In the third section, we give examples of various classes of operator-valued multishifts. We study circularity, analyticity and wandering subspace property of operator-valued multishifts in the fourth section. The last section deals with the study of function theoretic behaviour of operator-valued multishifts. In particular, we study the bounded point evaluation, reproducing kernel structure and the unitary equivalence of these multishifts. It turns out that the set of bounded point evaluations of operator-valued multishifts with invertible operator weights may be properly contained in the joint point spectrum of the adjoint of these multishifts (see Remark 5.4). This is in contrast with [24, Proposition 19].

We set below the notations used in posterior sections. For a set $X$ and positive integer $d, X^{d}$ stands for the $d$-fold Cartesian product of $X$. The symbol $\mathbb{N}, \mathbb{R}$ and $\mathbb{C}$ stand for the set of non-negative integers, field of the real numbers and the field of complex numbers, respectively. For $\alpha=\left(\alpha_{1}, \ldots, \alpha_{d}\right) \in \mathbb{N}^{d}$, we set $|\alpha|:=\sum_{j=1}^{d} \alpha_{j}$ and $\alpha !:=\prod_{j=1}^{d} \alpha_{j}$ !. We follow the convention that $\alpha \in \mathbb{N}^{d}$ is always understood as $\alpha=\left(\alpha_{1}, \ldots, \alpha_{d}\right)$. For $w=\left(w_{1}, \ldots, w_{d}\right) \in \mathbb{C}^{d}$ and $\alpha \in \mathbb{N}^{d}$, the complex conjugate $\bar{w} \in \mathbb{C}^{d}$ of $w$ is given by $\left(\bar{w}_{1}, \ldots, \bar{w}_{d}\right)$, while $w^{\alpha}$ denotes the complex number $\prod_{j=1}^{d} w_{j}^{\alpha_{j}}$. The symbol $\mathbb{T}^{d}$ denotes the $d$-torus in $\mathbb{C}^{d}$. A subset $\Omega$ of $\mathbb{C}^{d}$ is said to have polycircular symmetry if for every $\lambda=\left(\lambda_{1}, \ldots, \lambda_{d}\right) \in \mathbb{T}^{d}$ and $z=\left(z_{1}, \ldots, z_{d}\right) \in \Omega, \lambda \cdot z:=\left(\lambda_{1} z_{1}, \ldots, \lambda_{d} z_{d}\right) \in \Omega$. A connected subset of $\mathbb{C}^{d}$ is called Reinhardt if it has polycircular symmetry. Let $\mathcal{H}$ be a complex Hilbert space. If $F$ is a subset of $\mathcal{H}$, the closure of $F$ is denoted by $\bar{F}$, while the closed linear span of $F$ is denoted by $\bigvee\{x: x \in F\}$. If $\mathcal{M}$ is a subspace of $\mathcal{H}$, then $\operatorname{dim} \mathcal{M}$ denotes the Hilbert space dimension of $\mathcal{M}$. Let $\mathcal{B}(\mathcal{H})$ denote the unital Banach algebra of bounded linear operators on $\mathcal{H}$ whereas $\mathcal{G}(\mathcal{H}) \subseteq \mathcal{B}(\mathcal{H})$ denotes the set of invertible operators on $\mathcal{H}$. The multiplicative identity $I$ of $\mathcal{B}(\mathcal{H})$ is sometimes denoted by $I_{\mathcal{H}}$. The norm on $\mathcal{H}$ is denoted by $\|\cdot\|_{\mathcal{H}}$ and whenever there is no confusion likely, we remove the subscript $\mathcal{H}$ from $\|\cdot\|_{\mathcal{H}}$. If $T \in \mathcal{B}(\mathcal{H})$, then $\operatorname{ker}(T)$ denotes the kernel of $T$, the range of $T$ is denoted by $T(\mathcal{H})$ or $\operatorname{ran} T, T^{*}$ denotes the Hilbert space adjoint of $T$ and $r(T)$ denotes the spectral radius of $T$. An operator $T \in \mathcal{B}(\mathcal{H})$ is left-invertible if $T^{*} T$ is invertible in $\mathcal{B}(\mathcal{H})$. We say that $T \in \mathcal{B}(\mathcal{H})$ is analytic if $\bigcap_{n \in \mathbb{N}} T^{n}(\mathcal{H})=\{0\}$. By a commuting d-tuple $T=\left(T_{1}, \ldots, T_{d}\right)$ in $\mathcal{B}(\mathcal{H})$, we mean a collection of commuting operators $T_{1}, \ldots, T_{d}$ in $\mathcal{B}(\mathcal{H})$. For $\alpha \in \mathbb{N}^{d}$, we understand $T^{\alpha}$ as the operator $T_{1}^{\alpha_{1}} \cdots T_{d}^{\alpha_{d}}$, where we adhere to the convention that $A^{0}=I_{\mathcal{H}}$ for $A \in B(\mathcal{H})$. A commuting $d$-tuple $T=\left(T_{1}, \ldots, T_{d}\right)$ is said to be analytic if $\bigcap_{\alpha \in \mathbb{N}^{d}} T^{\alpha}(\mathcal{H})=\{0\}$. The notations $\sigma(T), \sigma_{e}(T), \sigma_{l}(T)$ and $\sigma_{p}(T)$ are reserved for the Taylor spectrum, joint essential spectrum, joint left-spectrum and joint point spectrum of a commuting $d$-tuple $T$ respectively. The Hilbert space adjoint of the commuting $d$-tuple $T=\left(T_{1}, \ldots, T_{d}\right)$ is the $d$-tuple $T^{*}=\left(T_{1}^{*}, \ldots, T_{d}^{*}\right)$, and the joint kernel $\bigcap_{j=1}^{d} \operatorname{ker} T_{j}$ of $T$ is denoted by $\operatorname{ker} T$. A commuting $d$-tuple $T=\left(T_{1}, \ldots, T_{d}\right)$ is said to be toral left invertible if $T_{j}^{*} T_{j}$ is invertible for each $j=1, \ldots, d$. Further, for $\lambda=\left(\lambda_{1}, \ldots, \lambda_{d}\right) \in \mathbb{C}^{d}$, by $T-\lambda$, we understand the $d$ tuple $\left(T_{1}-\lambda_{1} I_{\mathcal{H}}, \ldots, T_{d}-\lambda_{d} I_{\mathcal{H}}\right)$. A commuting $d$-tuple $T=\left(T_{1}, \ldots, T_{d}\right)$ is said to be a toral contraction (resp. a joint contraction) if $T_{j}^{*} T_{j} \leqslant I$ for every $j=1, \ldots, d$ (resp. $\left.\quad \sum_{j=1}^{d} T_{j}^{*} T_{j} \leqslant I\right)$. We say that $T=\left(T_{1}, \ldots, T_{d}\right)$ is a row contraction if $\sum_{j=1}^{d} T_{j} T_{j}^{*} \leqslant I$ and it is said to be joint expansion if $\sum_{j=1}^{d} T_{j}^{*} T_{j} \geqslant I$. 


\section{Operator-valued Multishift: Definition and basic properties}

Let $\left\{H_{\alpha}: \alpha \in \mathbb{N}^{d}\right\}$ be a multisequence of complex separable Hilbert spaces and let $\mathcal{H}=\oplus_{\alpha \in \mathbb{N}^{d}} H_{\alpha}$ be the orthogonal direct sum of $H_{\alpha}, \alpha \in \mathbb{N}^{d}$. Then $\mathcal{H}$ is a Hilbert space with respect to the following inner product:

$$
\langle x, y\rangle_{\mathcal{H}}=\sum_{\alpha \in \mathbb{N}^{d}}\left\langle x_{\alpha}, y_{\alpha}\right\rangle_{H_{\alpha}}, \quad x=\oplus_{\alpha \in \mathbb{N}^{d}} x_{\alpha}, y=\oplus_{\alpha \in \mathbb{N}^{d}} y_{\alpha} \in \mathcal{H}
$$

If $H_{\alpha}=H$ for all $\alpha \in \mathbb{N}^{d}$, then we denote $\mathcal{H}=\oplus_{\alpha \in \mathbb{N}^{d}} H$ by $\ell_{H}^{2}\left(\mathbb{N}^{d}\right)$. Let $\left\{A_{\alpha}^{(j)}\right.$ : $\left.\alpha \in \mathbb{N}^{d}, j=1, \ldots, d\right\}$ be a multisequence of bounded linear operators $A_{\alpha}^{(j)}: H_{\alpha} \rightarrow$ $H_{\alpha+\varepsilon_{j}}$. An operator-valued multishift $T$ on $\mathcal{H}=\oplus_{\alpha \in \mathbb{N}^{d}} H_{\alpha}$ with operator weights $\left\{A_{\alpha}^{(j)}: \alpha \in \mathbb{N}^{d}, j=1, \ldots, d\right\}$ is a $d$-tuple of operators $T_{1}, \ldots, T_{d}$ in $\mathcal{H}$ defined by

$$
\begin{gathered}
\mathcal{D}\left(T_{j}\right):=\left\{\oplus_{\alpha \in \mathbb{N}^{d}} x_{\alpha} \in \mathcal{H}: \sum_{\alpha \in \mathbb{N}^{d}}\left\|A_{\alpha}^{(j)} x_{\alpha}\right\|^{2}<\infty\right\}, \\
T_{j}\left(\oplus_{\alpha \in \mathbb{N}^{d}} x_{\alpha}\right):=\oplus_{\alpha \in \mathbb{N}^{d}} A_{\alpha-\varepsilon_{j}}^{(j)} x_{\alpha-\varepsilon_{j}}, \quad \oplus_{\alpha \in \mathbb{N}^{d}} x_{\alpha} \in \mathcal{D}\left(T_{j}\right), j=1, \ldots, d,
\end{gathered}
$$

where $\varepsilon_{j}$ is the $d$-tuple in $\mathbb{N}^{d}$ with 1 in the $j^{\text {th }}$ place and zeros elsewhere. If for $\alpha \in \mathbb{N}^{d}, \alpha_{j}=0$, then we interpret $A_{\alpha-\varepsilon_{j}}^{(j)}$ as a zero operator, $x_{\alpha-\varepsilon_{j}}$ as a zero vector and $H_{\alpha-\varepsilon_{j}}:=\{0\}$. In what follows, if we encounter a situation where $\alpha_{j}$, in $\left(\alpha_{1}, \ldots, \alpha_{d}\right)$, is negative for some $j \in\{1, \ldots, d\}$, then we set $H_{\alpha}=\{0\}$, $A_{\alpha}^{(j)}: H_{\alpha} \rightarrow H_{\alpha+\varepsilon_{j}}$ to be a zero operator and $x_{\alpha}$ as a zero vector.

Note that each $T_{j}, j=1, \ldots, d$, is a densely defined linear operator in $\mathcal{H}$. It follows from [18, Proposition 3.1] that $T_{j}$ is bounded if and only if

$$
\sup _{\alpha \in \mathbb{N}^{d}}\left\|A_{\alpha}^{(j)}\right\|<\infty .
$$

Further, $T_{i}$ commutes with $T_{j}$ if and only if

$$
A_{\alpha+\varepsilon_{j}}^{(i)} A_{\alpha}^{(j)}=A_{\alpha+\varepsilon_{i}}^{(j)} A_{\alpha}^{(i)} \text { for all } \alpha \in \mathbb{N}^{d} .
$$

That is, the following diagram commutes:

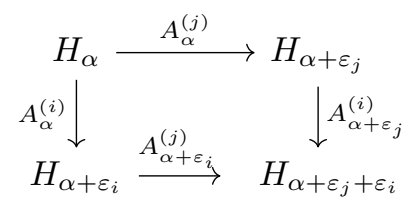

We refer to $T$ as commuting operator-valued multishift if the operator weights satisfy (1) and (2). The proof of the following proposition is a routine verification. We leave it for the interested readers.

Proposition 2.1. Let $T=\left(T_{1}, \ldots, T_{d}\right)$ be a commuting operator-valued multishift on $\mathcal{H}=\oplus_{\alpha \in \mathbb{N}^{d}} H_{\alpha}$ with operator weights $\left\{A_{\alpha}^{(j)}: \alpha \in \mathbb{N}^{d}, j=1, \ldots, d\right\}$. Then

$$
T_{j}^{*}\left(\oplus_{\alpha \in \mathbb{N}^{d}} x_{\alpha}\right)=\oplus_{\alpha \in \mathbb{N}^{d}} A_{\alpha}^{(j) *} x_{\alpha+\varepsilon_{j}}, \quad \oplus_{\alpha \in \mathbb{N}^{d}} x_{\alpha} \in \mathcal{H}, j=1, \ldots, d .
$$

Further, the joint kernel $\operatorname{ker} T^{*}$ of $T^{*}$ is given by

$$
\operatorname{ker} T^{*}=H_{0} \oplus \bigoplus_{\alpha \in \mathbb{N}^{d} \backslash\{0\}} \operatorname{ker} A_{\alpha}^{*},
$$

where $\operatorname{ker} A_{\alpha}^{*}=\bigcap_{j=1}^{d} \operatorname{ker} A_{\alpha-\varepsilon_{j}}^{(j) *}, \alpha \in \mathbb{N}^{d} \backslash\{0\}$.

The following proposition shows that there is no non-zero normal operatorvalued multishift. 
Proposition 2.2. Let $T=\left(T_{1}, \ldots, T_{d}\right)$ be a commuting operator-valued multishift on $\mathcal{H}=\oplus_{\alpha \in \mathbb{N}^{d}} H_{\alpha}$ with operator weights $\left\{A_{\alpha}^{(j)}: \alpha \in \mathbb{N}^{d}, j=1, \ldots, d\right\}$. Then for each $j=1, \ldots, d, T_{j}$ is normal if and only if $T_{j}$ is zero.

Proof. Fix $j \in\{1, \ldots, d\}$. Clearly, if $T_{j}$ is zero, then it is normal. Suppose that $T_{j}$ is normal. Then for all $\oplus_{\alpha \in \mathbb{N}^{d}} x_{\alpha} \in \mathcal{H}$, we get

$$
\oplus_{\alpha \in \mathbb{N}^{d}} A_{\alpha}^{(j) *} A_{\alpha}^{(j)} x_{\alpha}=\oplus_{\alpha \in \mathbb{N}^{d}} A_{\alpha-\varepsilon_{j}}^{(j)} A_{\alpha-\varepsilon_{j}}^{(j) *} x_{\alpha},
$$

which in turn implies that

$$
A_{\alpha}^{(j) *} A_{\alpha}^{(j)}=A_{\alpha-\varepsilon_{j}}^{(j)} A_{\alpha-\varepsilon_{j}}^{(j) *} \text { for all } \alpha \in \mathbb{N}^{d} .
$$

It follows from (4) that for $\alpha=\left(\alpha_{1}, \ldots, \alpha_{d}\right) \in \mathbb{N}^{d}, A_{\alpha}^{(j)}=0$ if $\alpha_{j}=0$. To show that $A_{\alpha}^{(j)}=0$ for all $\alpha \in \mathbb{N}^{d}$, we apply mathematical induction on $\alpha_{j}$. Clearly, the induction hypothesis holds for $\alpha_{j}=0$. Suppose that for $k \in \mathbb{N}, A_{\alpha}^{(j)}=0$ for all $\alpha \in \mathbb{N}^{d}$ with $\alpha_{j}=k$. Let $\beta \in \mathbb{N}^{d}$ be such that $\beta_{j}=k+1$. Then from (4), we get

$$
A_{\beta}^{(j) *} A_{\beta}^{(j)}=A_{\beta-\varepsilon_{j}}^{(j)} A_{\beta-\varepsilon_{j}}^{(j) *} .
$$

Since $\beta_{j}-1=k$, it follows from the induction hypothesis that $A_{\beta-\varepsilon_{j}}^{(j)}=0$ and hence $A_{\beta}^{(j) *} A_{\beta}^{(j)}=0$ which in turn implies that $A_{\beta}^{(j)}=0$. Thus $T_{j}$ must be zero.

The following proposition gives explicit formula for the moments of a commuting operator-valued multishift. We will see later that these moments play a central role in the study of function theoretic behaviour of these multishifts.

Proposition 2.3. Let $T=\left(T_{1}, \ldots, T_{d}\right)$ be a commuting operator-valued multishift on $\mathcal{H}=\oplus_{\alpha \in \mathbb{N}^{d}} H_{\alpha}$ with operator weights $\left\{A_{\alpha}^{(j)}: \alpha \in \mathbb{N}^{d}, j=1, \ldots, d\right\}$. For each $\alpha, \beta \in \mathbb{N}^{d}$, define

$$
B(\alpha, \beta):=A^{(1)}\left(\alpha, \beta_{1}\right) A^{(2)}\left(\alpha-\beta_{1} \varepsilon_{1}, \beta_{2}\right) \cdots A^{(d)}\left(\alpha-\sum_{l=1}^{d-1} \beta_{l} \varepsilon_{l}, \beta_{d}\right),
$$

where for each $j \in\{1, \ldots, d\}, A^{(j)}(\alpha, k): H_{\alpha-k \varepsilon_{j}} \rightarrow H_{\alpha}$ is given by

$$
A^{(j)}(\alpha, k):= \begin{cases}A_{\alpha-\varepsilon_{j}}^{(j)} \cdots A_{\alpha-k \varepsilon_{j}}^{(j)} & \text { if } k \geqslant 1, \\ I_{H_{\alpha}} & \text { if } k=0 .\end{cases}
$$

Then for all $\alpha, \beta \in \mathbb{N}^{d}$, the following statements are true:

(i) $A_{\alpha}^{(j)} A^{(l)}(\alpha, k)=A^{(l)}\left(\alpha+\varepsilon_{j}, k\right) A_{\alpha-k \varepsilon_{l}}^{(j)}$ for all $j, l=1, \ldots, d$ and $k \in \mathbb{N}$.

(ii) $A_{\alpha}^{(j)} B(\alpha, \beta)=B\left(\alpha+\varepsilon_{j}, \beta+\varepsilon_{j}\right)$ for all $j=1, \ldots, d$.

(iii) $T^{\beta}\left(\oplus_{\alpha \in \mathbb{N}^{d}} x_{\alpha}\right)=\oplus_{\alpha \in \mathbb{N}^{d}} B(\alpha, \beta) x_{\alpha-\beta}, \oplus_{\alpha \in \mathbb{N}^{d}} x_{\alpha} \in \mathcal{H}$.

(iv) $T^{* \beta}\left(\oplus_{\alpha \in \mathbb{N}^{d}} x_{\alpha}\right)=\oplus_{\alpha \in \mathbb{N}^{d}} C(\alpha, \beta) x_{\alpha+\beta}, \oplus_{\alpha \in \mathbb{N}^{d}} x_{\alpha} \in \mathcal{H}$, where

$$
C(\alpha, \beta)=C^{(1)}\left(\alpha, \beta_{1}\right) C^{(2)}\left(\alpha+\beta_{1} \varepsilon_{1}, \beta_{2}\right) \cdots C^{(d)}\left(\alpha+\sum_{l=1}^{d-1} \beta_{l} \varepsilon_{l}, \beta_{d}\right)
$$

and for $j \in\{1, \ldots, d\}$,

$$
C^{(j)}(\alpha, k)= \begin{cases}A_{\alpha}^{(j) *} \cdots A_{\alpha+(k-1) \varepsilon_{j}}^{(j) *} & \text { if } k \geqslant 1 \\ I_{H_{\alpha}} & \text { if } k=0 .\end{cases}
$$

(v) $T^{* \beta} T^{\beta}\left(\oplus_{\alpha \in \mathbb{N}^{d}} x_{\alpha}\right)=\oplus_{\alpha \in \mathbb{N}^{d}} C(\alpha, \beta) B(\alpha+\beta, \beta) x_{\alpha}, \oplus_{\alpha \in \mathbb{N}^{d}} x_{\alpha} \in \mathcal{H}$.

Note: For the sake of convenience, we write $B_{\alpha}$ in place of $B(\alpha, \alpha)$. 
Proof. The equality in (i) follows from the repeated applications of commuting condition (2). Indeed, if $k=0$, then the equality holds trivially for all $\alpha \in \mathbb{N}^{d}$. Let $\alpha \in \mathbb{N}^{d}, j, l \in\{1, \ldots, d\}$ and $k \geqslant 1$. Then

$$
\begin{aligned}
A_{\alpha}^{(j)} A^{(l)}(\alpha, k) & =A_{\alpha}^{(j)} A_{\alpha-\varepsilon_{l}}^{(l)} \cdots A_{\alpha-k \varepsilon_{l}}^{(l)} \stackrel{\text { (2) }}{=} A_{\alpha+\epsilon_{j}-\epsilon_{l}}^{(l)} A_{\alpha-\varepsilon_{l}}^{(j)} \cdots A_{\alpha-k \varepsilon_{l}}^{(l)} \\
& \stackrel{(2)}{=} \cdots \stackrel{(2)}{=} A_{\alpha+\epsilon_{j}-\epsilon_{l}}^{(l)} \cdots A_{\alpha+\varepsilon_{j}-k \varepsilon_{l}}^{(l)} A_{\alpha-k \varepsilon_{l}}^{(j)}=A^{(l)}\left(\alpha+\varepsilon_{j}, k\right) A_{\alpha-k \varepsilon_{l}}^{(j)} .
\end{aligned}
$$

To see (ii), let $\alpha, \beta \in \mathbb{N}^{d}$ and $j \in\{1, \ldots, d\}$. Then

$$
\begin{aligned}
& A_{\alpha}^{(j)} B(\alpha, \beta)=A_{\alpha}^{(j)} A^{(1)}\left(\alpha, \beta_{1}\right) A^{(2)}\left(\alpha-\beta_{1} \varepsilon_{1}, \beta_{2}\right) \cdots A^{(d)}\left(\alpha-\sum_{l=1}^{d-1} \beta_{l} \varepsilon_{l}, \beta_{d}\right) \\
& \stackrel{(\mathrm{i})}{=} A^{(1)}\left(\alpha+\varepsilon_{j}, \beta_{1}\right) A_{\alpha-\beta_{1} \varepsilon_{1}}^{(j)} \cdots A^{(d)}\left(\alpha-\sum_{l=1}^{d-1} \beta_{l} \varepsilon_{l}, \beta_{d}\right) .
\end{aligned}
$$

Continuing in this way, the right hand side of above expression becomes

$$
A^{(1)}\left(\alpha+\varepsilon_{j}, \beta_{1}\right) \cdots A^{(j)}\left(\alpha-\sum_{l=1}^{j-1} \beta_{l} \varepsilon_{l}+\varepsilon_{j}, \beta_{j}\right) A_{\alpha-\sum_{l=1}^{j} \beta_{l} \varepsilon_{l}}^{(j)} \cdots A^{(d)}\left(\alpha-\sum_{l=1}^{d-1} \beta_{l} \varepsilon_{l}, \beta_{d}\right) .
$$

Note that

$$
A^{(j)}\left(\alpha-\sum_{l=1}^{j-1} \beta_{l} \varepsilon_{l}+\varepsilon_{j}, \beta_{j}\right) A_{\alpha-\sum_{l=1}^{j} \beta_{l} \varepsilon_{l}}^{(j)}=A^{(j)}\left(\alpha-\sum_{l=1}^{j-1} \beta_{l} \varepsilon_{l}+\varepsilon_{j}, \beta_{j}+1\right) .
$$

Thus we get (ii).

We prove (iii) by induction on $|\beta|, \beta \in \mathbb{N}^{d}$. If $|\beta|=0$, then $\beta=0$ and hence the equality in (iii) holds trivially. Suppose that for some $n \in \mathbb{N}$, it holds for all $\beta \in \mathbb{N}^{d}$ with $|\beta|=n$. Let $\gamma \in \mathbb{N}^{d}$ be such that $|\gamma|=n+1$. Then $\gamma=\beta+\varepsilon_{j}$ for some $j \in\{1, \ldots, d\}$ and $|\beta|=n$. Now for $\oplus_{\alpha \in \mathbb{N}^{d}} x_{\alpha} \in \mathcal{H}$, we get

$$
\begin{aligned}
T^{\gamma}\left(\oplus_{\alpha \in \mathbb{N}^{d}} x_{\alpha}\right) & =T_{j} T^{\beta}\left(\oplus_{\alpha \in \mathbb{N}^{d}} x_{\alpha}\right)=T_{j}\left(\oplus_{\alpha \in \mathbb{N}^{d}} B(\alpha, \beta) x_{\alpha-\beta}\right) \\
& =\oplus_{\alpha \in \mathbb{N}^{d}} A_{\alpha-\varepsilon_{j}}^{(j)} B\left(\alpha-\varepsilon_{j}, \beta\right) x_{\alpha-\varepsilon_{j}-\beta} \\
& \stackrel{(\mathrm{ii})}{=} \oplus_{\alpha \in \mathbb{N}^{d}} B(\alpha, \gamma) x_{\alpha-\gamma}
\end{aligned}
$$

This completes the proof of (iii). The proof of (iv) goes along the lines of that of (iii) while (v) follows from (iii) and (iv).

Corollary 2.4. Let $T=\left(T_{1}, \ldots, T_{d}\right)$ be a commuting operator-valued multishift on $\mathcal{H}=\bigoplus_{\alpha \in \mathbb{N}^{d}} H_{\alpha}$ with operator weights $\left\{A_{\alpha}^{(j)}: \alpha \in \mathbb{N}^{d}, j=1, \ldots, d\right\}$. For $k \in \mathbb{N}$, let $D^{(k)}=\left(T_{1}^{* k}, \ldots, T_{d}^{* k}\right)$. Then $\bigcup_{k \in \mathbb{N}} \operatorname{ker} D^{(k)}$ is a dense subspace of $\mathcal{H}$.

Proof. Note that $\left\{\operatorname{ker} D^{(k)}\right\}_{k \in \mathbb{N}}$ is an increasing sequence of subspaces of $\mathcal{H}$ and hence $\cup_{k \in \mathbb{N}} \operatorname{ker} D^{(k)}$ is a subspace of $\mathcal{H}$. For each $\alpha \in \mathbb{N}^{d}$, define

$$
M_{\alpha}:=\left\{\oplus_{\beta \in \mathbb{N}^{d}} x_{\beta} \in \mathcal{H}: x_{\beta}=0 \text { if } \beta \neq \alpha\right\} .
$$

Fix $k \geqslant 1$ and $j \in\{1, \ldots, d\}$. Let $\alpha \in \mathbb{N}^{d}$ be such that $|\alpha|<k$. Then by substituting $\beta=k \varepsilon_{j}$ in Proposition 2.3(iv), we see that $M_{\alpha} \subseteq \operatorname{ker} T_{j}^{* k}$. Consequently, $M_{\alpha} \subseteq$ $\operatorname{ker} D^{(k)}$. Thus $\bigcup_{k \in \mathbb{N}} \operatorname{ker} D^{(k)}$ is a dense subspace of $\mathcal{H}$.

REMARK 2.5. It follows from the above corollary that $M_{0} \subseteq \operatorname{ker} T^{*}$. Thus $0 \in \sigma_{p}\left(T^{*}\right) \subseteq \sigma\left(T^{*}\right)$.

It is noted in [23, Pg. 7] that using [23, Proposition 2.1.12(vi), (viii)] a weighted shift on an arbitrary directed tree can be realized as an operator-valued weighted shift with underlying directed tree being $\mathbb{N}, \mathbb{Z}, \mathbb{N}_{-}$(set of all negative integers) or $\{0, \ldots, n\}$ for some $n \in \mathbb{N}$. Following the idea of [23, Proposition 2.1.12] and the 
notion of depth introduced in [5. Definition 2.1.11], in what follows, it turns out that the class of commuting multishifts on directed Cartesian product of rooted directed trees lies in that of commuting operator-valued multishifts. The reader is referred to [5, Chapters 2,3] for the definition and basic properties of multishifts on directed Cartesian product of rooted directed trees (see also $\mathbf{2 3}$ for general theory of weighted shifts on directed trees).

Proposition 2.6. Let $\mathscr{T}=(V, \mathcal{E})$ be the directed Cartesian product of rooted directed trees $\mathscr{T}_{1}, \ldots, \mathscr{T}_{d}$ and let $S_{\boldsymbol{\lambda}}=\left(S_{1}, \ldots, S_{d}\right)$ be a commuting multishift on $\mathscr{T}$ with weights $\left\{\lambda_{v}^{(j)}: v \in V \backslash\{\right.$ root $\left.\}, j=1, \ldots, d\right\}$. Then $S_{\boldsymbol{\lambda}}$ is unitarily equivalent to a commuting operator-valued multishift.

Proof. For each $\alpha \in \mathbb{N}^{d}$, let

$$
V_{\alpha}:=\left\{v \in V: \mathrm{d}_{v}=\alpha\right\} \text { and } H_{\alpha}:=\ell^{2}\left(V_{\alpha}\right),
$$

where $\mathrm{d}_{v}$ is the depth of $v$ in $\mathscr{T}$ (see [5, Definition 2.1.11]). Note that $V=\bigsqcup_{\alpha \in \mathbb{N}^{d}} V_{\alpha}$ (see [5. Lemma 2.1.10(vi)]). Set $\mathcal{H}:=\oplus_{\alpha \in \mathbb{N}^{d}} H_{\alpha}$. Then there is a natural isometry from $\ell^{2}(V)$ into $\mathcal{H}$. Indeed, let $x=\sum_{v \in V}\left\langle x, e_{v}\right\rangle e_{v} \in \ell^{2}(V)$, then

$$
x=\sum_{v \in V}\left\langle x, e_{v}\right\rangle e_{v}=\sum_{\alpha \in \mathbb{N}^{d}} \sum_{v \in V_{\alpha}}\left\langle x, e_{v}\right\rangle e_{v}=\sum_{\alpha \in \mathbb{N}^{d}} x_{\alpha},
$$

where $x_{\alpha}=\sum_{v \in V_{\alpha}}\left\langle x, e_{v}\right\rangle e_{v} \in H_{\alpha}$. Here for $W \subseteq V$, we identify $\ell^{2}(W)$ as a subspace of $\ell^{2}(V)$. Now define $U: \ell^{2}(V) \rightarrow \mathcal{H}$ by

$$
U x=\oplus_{\alpha \in \mathbb{N}^{d}} x_{\alpha}, \quad x \in \ell^{2}(V) .
$$

It is easy to see that $U$ is unitary. Now for each $\alpha \in \mathbb{N}^{d}$ and $j \in\{1, \ldots, d\}$, consider the linear operator $A_{\alpha}^{(j)}: H_{\alpha} \rightarrow H_{\alpha+\varepsilon_{j}}$ given by

$$
A_{\alpha}^{(j)} x=S_{j} x, \quad x \in H_{\alpha} .
$$

Clearly, $A_{\alpha}^{(j)}$ is bounded for each $\alpha \in \mathbb{N}^{d}$ and $j \in\{1, \ldots, d\}$. Let $T=\left(T_{1}, \ldots, T_{d}\right)$ be the operator-valued multishift on $\mathcal{H}$ with operator weights $\left\{A_{\alpha}^{(j)}: \alpha \in \mathbb{N}^{d}, j=\right.$ $1, \ldots, d\}$. Since $S_{\boldsymbol{\lambda}}$ is commuting, it follows that $T$ is also commuting. Further, for $x \in \ell^{2}(V)$ and $j \in\{1, \ldots, d\}$, we have

$$
\begin{aligned}
T_{j} U x & =T_{j}\left(\oplus_{\alpha \in \mathbb{N}^{d}} x_{\alpha}\right)=\oplus_{\alpha \in \mathbb{N}^{d}} A_{\alpha-\varepsilon_{j}}^{(j)} x_{\alpha-\varepsilon_{j}}=\oplus_{\alpha \in \mathbb{N}^{d}} S_{j} x_{\alpha-\varepsilon_{j}} \\
& =U\left(\sum_{\alpha \in \mathbb{N}^{d}} S_{j} x_{\alpha-\varepsilon_{j}}\right)=U S_{j}\left(\sum_{\alpha \in \mathbb{N}^{d}} x_{\alpha-\varepsilon_{j}}\right)=U S_{j} x .
\end{aligned}
$$

This completes the proof.

A natural question arises here whether a commuting operator-valued multishift can be looked upon as a multishift on a directed Cartesian product of rooted directed trees. We do not know the answer of this question in more than one variable, but in case $d=1$, there are some sufficient conditions which ensure an affirmative answer of this question.

Proposition 2.7. Let $T$ be an operator-valued unilateral weighted shift on $\mathcal{H}=\oplus_{n \in \mathbb{N}} H_{n}$ with operator weights $\left\{A_{n}: n \in \mathbb{N}, j=1, \ldots, d\right\}$. Suppose that $\operatorname{dim} H_{0} \geqslant 1$ and for each $n \in \mathbb{N}$, there exists an orthonormal basis $B_{n}$ of $H_{n}$ with the following property: For each $x \in B_{n}$ there exists a subset $W_{x}$ of $B_{n+1}$ such that

(a) $B_{n+1}=\bigsqcup_{x \in B_{n}} W_{x}$,

(b) $\left\langle A_{n} x, y\right\rangle \neq 0$ for all $y \in W_{x}$ and $A_{n} x=\sum_{y \in W_{x}}\left\langle A_{n} x, y\right\rangle y$.

Then $T$ is unitarily equivalent to direct sum of $\operatorname{dim} H_{0}$ number of weighted shifts on rooted directed trees. 
Proof. Set $V:=\cup_{n \in \mathbb{N}} B_{n}$ and declare that for $x \in B_{n}$, Chi $(x)=W_{x}$. This determines countably many disjoint rooted directed trees with roots being the elements of $B_{0}$. For $x \in B_{n}, n \geqslant 1$, set $\lambda_{x}:=\left\langle A_{n-1} \operatorname{par}(x), x\right\rangle_{H_{n}}$ and consider the weight system $\lambda=\left\{\lambda_{x}: x \in V \backslash B_{0}\right\}$ of non-zero complex numbers. Let $\ell^{2}(V)$ be the Hilbert space of square summable complex-valued functions on $V$ with standard inner product. Note that the set $\left\{e_{x}: x \in V\right\}$ of characteristic functions of the singletons form an orthonormal basis of $\ell^{2}(V)$. Then the weighted shift operator $S_{\lambda}$ on $\ell^{2}(V)$ is a bounded linear operator. Let $U$ be the unitary operator from $\ell^{2}(V)$ onto $\mathcal{H}$ such that

$$
U e_{x}=e_{n} \otimes x, \quad x \in V,
$$

where $x \in B_{n}, n \in \mathbb{N}$, and

$$
e_{n} \otimes x:=\oplus_{k=0}^{\infty} x_{k}= \begin{cases}x & \text { if } k=n, \\ 0 & \text { otherwise }\end{cases}
$$

Then for $x \in B_{n}, n \in \mathbb{N}$, we have

$$
\begin{aligned}
U S_{\lambda} e_{x} & =U \sum_{y \in \operatorname{Chi}(x)} \lambda_{y} e_{y}=\sum_{y \in W_{x}}\left\langle A_{n} \operatorname{par}(y), y\right\rangle\left(e_{n+1} \otimes y\right) \\
& =e_{n+1} \otimes\left(\sum_{y \in W_{x}}\left\langle A_{n} \operatorname{par}(y), y\right\rangle y\right)=e_{n+1} \otimes A_{n} x=T U e_{x} .
\end{aligned}
$$

This shows that $T$ is unitarily equivalent to $S_{\lambda}$. Further, it is easy to see that

$$
V=\bigsqcup_{x \in B_{0}} V_{x}
$$

where for $x \in B_{0}$,

$$
V_{x}:=\bigcup_{n \in \mathbb{N}} \operatorname{Chi}^{\langle n\rangle}(x)=\{x\} \cup W_{x} \cup\left(\bigcup_{y \in W_{x}} W_{y}\right) \cup \cdots .
$$

Thus we get $\ell^{2}(V)=\oplus_{x \in B_{0}} \ell^{2}\left(V_{x}\right)$ and each $\ell^{2}\left(V_{x}\right)$ is a reducing subspace of $S_{\lambda}$. Also note that for each $x \in B_{0}, V_{x}$ is a rooted directed tree with root $x$. Hence $S_{\lambda}=\oplus_{x \in B_{0}} S_{x}$, where $S_{x}:=\left.S_{\lambda}\right|_{\ell^{2}\left(V_{x}\right)}$ is a weighted shift on the rooted directed tree $V_{x}$. This completes the proof of the proposition.

As an immediate consequence of the preceding proposition, we get the following corollary.

Corollary 2.8. Under the assumptions of Proposition 2.7. if $\operatorname{dim} H_{0}=1$, then $T$ is unitarily equivalent to a weighted shift on a rooted directed tree.

\section{Examples}

We have already seen in Proposition 2.6 that a large class of examples of operator-valued multishifts is supplied by the multishifts on directed Cartesian product of rooted directed trees. In this section, we exhibit few more classes of examples of commuting operator-valued multishifts. We begin with the following well-known example.

EXAMPLE 3.1. Let $\left\{w_{\alpha}^{(j)}: \alpha \in \mathbb{N}^{d}, j=1, \ldots, d\right\}$ be a multisequence of nonzero complex numbers such that $\sup _{\alpha \in \mathbb{N}^{d}}\left|w_{\alpha}^{(j)}\right|<\infty$ and $w_{\alpha+\varepsilon_{i}}^{(j)} w_{\alpha}^{(i)}=w_{\alpha+\varepsilon_{j}}^{(i)} w_{\alpha}^{(j)}$ for all $\alpha \in \mathbb{N}^{d}, i, j=1, \ldots, d$. Let $\mathcal{H}=\ell_{\mathbb{C}}^{2}\left(\mathbb{N}^{d}\right)$. Set $A_{\alpha}^{(j)}:=w_{\alpha}^{(j)} I_{\mathbb{C}}$ for all $\alpha \in \mathbb{N}^{d}$ and $j=1, \ldots, d$. Then the commuting operator-valued multishift $T=\left(T_{1}, \ldots, T_{d}\right)$ with operator weights $\left\{A_{\alpha}^{(j)}: \alpha \in \mathbb{N}^{d}, j=1, \ldots, d\right\}$ is commonly known as classical multishift [24]. 
The following proposition gives a recipe to construct a number of examples of various classes of commuting operator-valued multishifts.

Proposition 3.2. Let $\left\{w_{\alpha}^{(j)}: \alpha \in \mathbb{N}^{d}, j=1, \ldots, d\right\}$ be a multisequence of nonzero complex numbers such that $\sup _{\alpha \in \mathbb{N}^{d}}\left|w_{\alpha}^{(j)}\right|<\infty$ and $w_{\alpha+\varepsilon_{i}}^{(j)} w_{\alpha}^{(i)}=w_{\alpha+\varepsilon_{j}}^{(i)} w_{\alpha}^{(j)}$ for all $\alpha \in \mathbb{N}^{d}, i, j=1, \ldots, d$. Let $H$ be a complex separable Hilbert space and let $\Phi: \mathbb{N} \rightarrow \mathcal{B}(H)$ be a bounded function. For all $\alpha \in \mathbb{N}^{d}$ and $j \in\{1, \ldots, d\}$, set

$$
A_{\alpha}^{(j)}:=w_{\alpha}^{(j)} \Phi(|\alpha|)
$$

Consider the operator-valued multishift $T=\left(T_{1}, \ldots, T_{d}\right)$ on $\mathcal{H}=\ell_{H}^{2}\left(\mathbb{N}^{d}\right)$ with operator weights $\left\{A_{\alpha}^{(j)}: \alpha \in \mathbb{N}^{d}, j=1, \ldots, d\right\}$. For all $\alpha \in \mathbb{N}^{d}$, set

$$
w_{\alpha}:=\left(w_{\alpha}^{(1)}, \ldots, w_{\alpha}^{(d)}\right), \quad \tilde{w}_{\alpha}:=\left(w_{\alpha-\varepsilon_{1}}^{(1)}, \ldots, w_{\alpha-\varepsilon_{d}}^{(d)}\right),
$$

where we follow the convention that for $j \in\{1, \ldots, d\}, w_{\alpha}^{(j)}=0$ whenever $\alpha_{k}<0$ for some $k \in\{1, \ldots, d\}$. Then the following statements hold:

(i) $T$ is a commuting operator-valued multishift.

(ii) $T$ is a joint contraction if and only if for all $\alpha \in \mathbb{N}^{d}$, the Hilbert space operator $w_{\alpha} \otimes \Phi(|\alpha|): \mathbb{C}^{d} \otimes H \rightarrow H$ is a contraction.

(iii) $T$ is a row contraction if and only if for all $\alpha \in \mathbb{N}^{d}$ with $|\alpha| \geqslant 1$, the Hilbert space operator $\tilde{w}_{\alpha} \otimes \Phi(|\alpha|-1): \mathbb{C}^{d} \otimes H \rightarrow H$ is a contraction.

(iv) $T$ is a joint expansion if and only if for all $\alpha \in \mathbb{N}^{d}$, the Hilbert space operator $w_{\alpha} \otimes \Phi(|\alpha|): \mathbb{C}^{d} \otimes H \rightarrow H$ is an expansion.

ProOF. It follows from the hypotheses that $\sup _{\alpha \in \mathbb{N}^{d}}\left\|A_{\alpha}^{(j)}\right\|<\infty, j=1, \ldots, d$. For $i, j \in\{1, \ldots, d\}$ and $\alpha \in \mathbb{N}^{d}$, we have

$A_{\alpha+\varepsilon_{i}}^{(j)} A_{\alpha}^{(i)}=w_{\alpha+\epsilon_{i}}^{(j)} w_{\alpha}^{(i)} \Phi(|\alpha|+1) \Phi(|\alpha|)=w_{\alpha+\varepsilon_{j}}^{(i)} w_{\alpha}^{(j)} \Phi(|\alpha|+1) \Phi(|\alpha|)=A_{\alpha+\varepsilon_{j}}^{(i)} A_{\alpha}^{(j)}$.

Now (i) follows from the commuting condition (2).

Note that the commuting operator-valued multishift $T$ is a joint contraction if and only if for all $\alpha \in \mathbb{N}^{d}, \sum_{j=1}^{d} A_{\alpha}^{(j) *} A_{\alpha}^{(j)} \leqslant I_{H}$. Fix $\alpha \in \mathbb{N}^{d}$. Then we get

$$
I_{H} \geqslant \sum_{j=1}^{d} A_{\alpha}^{(j) *} A_{\alpha}^{(j)}=\left(\sum_{j=1}^{d}\left|w_{\alpha}^{(j)}\right|^{2}\right) \Phi(|\alpha|)^{*} \Phi(|\alpha|)=\left\|w_{\alpha}\right\| \Phi(|\alpha|)^{*}\left\|w_{\alpha}\right\| \Phi(|\alpha|) .
$$

Thus $\left\|w_{\alpha}\right\|\|\Phi(|\alpha|)\| \leqslant 1$. Hence $w_{\alpha} \otimes \Phi(|\alpha|)$ is a contraction. This verifies (ii).

To see (iii), note that $T$ is a row contraction if and only if for all $\alpha \in \mathbb{N}^{d}$, $\sum_{j=1}^{d} A_{\alpha-\varepsilon_{j}}^{(j)} A_{\alpha-\varepsilon_{j}}^{(j) *} \leqslant I_{H}$. Fix $\alpha \in \mathbb{N}^{d}$ with $|\alpha| \geqslant 1$. Then we get

$$
\begin{aligned}
I_{H} & \geqslant \sum_{j=1}^{d} A_{\alpha-\varepsilon_{j}}^{(j)} A_{\alpha-\varepsilon_{j}}^{(j) *}=\left(\sum_{j=1}^{d}\left|w_{\alpha-\varepsilon_{j}}^{(j)}\right|^{2}\right) \Phi(|\alpha|-1)^{*} \Phi(|\alpha|-1) \\
& =\left\|\tilde{w}_{\alpha}\right\| \Phi(|\alpha|-1)^{*}\left\|\tilde{w}_{\alpha}\right\| \Phi(|\alpha|-1) .
\end{aligned}
$$

Thus $\left\|\tilde{w}_{\alpha}\right\|\|\Phi(|\alpha|-1)\| \leqslant 1$. Hence $\tilde{w}_{\alpha} \otimes \Phi(|\alpha|-1)$ is a contraction. This proves (iii). The proof of (iv) goes similarly along the lines of the proof of (ii).

We below give an example of a commuting operator-valued multishifts with invertible operator weights which is a row contraction but not a joint contraction. 
ExAmple 3.3. Consider the system $\left\{A_{\alpha}^{(j)}: \alpha \in \mathbb{N}^{d}, j=1, \ldots, d\right\}$ of $2 \times 2$ matrices given by

$$
\begin{aligned}
& A_{\alpha}^{(j)}:=\frac{1}{2} \sqrt{\frac{\alpha j+1}{|\alpha|+1}}\left(\begin{array}{cc}
\sqrt{\frac{|\alpha|+2}{|\alpha|+3}}+\sqrt{\frac{|\alpha|}{|\alpha|+1}} & \sqrt{\frac{|\alpha|+2}{|\alpha|+3}}-\sqrt{\frac{|\alpha|}{|\alpha|+1}} \\
\sqrt{\frac{|\alpha|+2}{|\alpha|+3}}-\sqrt{\frac{|\alpha|}{|\alpha|+1}} & \sqrt{\frac{|\alpha|+2}{|\alpha|+3}}+\sqrt{\frac{|\alpha|}{|\alpha|+1}}
\end{array}\right), \quad \alpha \in \mathbb{N}^{d} \backslash\{0\}, \\
& A_{0}^{(j)}:=\frac{1}{2 \sqrt{3}}\left(\begin{array}{cc}
\sqrt{3}+1 & 1-\sqrt{3} \\
1-\sqrt{3} & \sqrt{3}+1
\end{array}\right) .
\end{aligned}
$$

It is easy to see that the operator-valued multishift $T=\left(T_{1}, \ldots, T_{d}\right)$ on $\mathcal{H}=\ell_{\mathbb{C}^{2}}^{2}\left(\mathbb{N}^{d}\right)$ with operator weights $\left\{A_{\alpha}^{(j)}: \alpha \in \mathbb{N}^{d}, j=1, \ldots, d\right\}$ is commuting. Further, $T$ is a row contraction and $T$ is a joint contraction if and only if $d=1$.

To see this, first observe that

$$
A_{\alpha}^{(j)}=w_{\alpha}^{(j)}\left(\begin{array}{ll}
\phi(|\alpha|)+\psi(|\alpha|) & \phi(|\alpha|)-\psi(|\alpha|) \\
\phi(|\alpha|)-\psi(|\alpha|) & \phi(|\alpha|)+\psi(|\alpha|)
\end{array}\right)
$$

where $w_{\alpha}^{(j)}:=\frac{1}{2} \sqrt{\frac{\alpha_{j}+1}{|\alpha|+1}}, \phi(n):=\sqrt{\frac{n+2}{n+3}}$ and $\psi(n):=\sqrt{\frac{n}{n+1}}$ for all $\alpha \in \mathbb{N}^{d} \backslash\{0\}$, $j \in\{1, \ldots, d\}$ and $n \in \mathbb{N}$. Now fix $\alpha \in \mathbb{N}^{d}$ with $|\alpha| \geqslant 1$. Then we have

$$
\left\|\tilde{w}_{\alpha}\right\|^{2}=\left\{\begin{array}{ll}
\frac{1}{4} \sum_{j=1}^{d} \frac{\alpha_{j}}{|\alpha|}=\frac{1}{4} & \text { if }|\alpha| \geqslant 2, \\
\frac{1}{(2 \sqrt{3})^{2}} & \text { if }|\alpha|=1,
\end{array} \quad\|\Phi(|\alpha|-1)\|= \begin{cases}2 \phi(|\alpha|-1) & \text { if }|\alpha| \geqslant 2, \\
2 \sqrt{3} & \text { if }|\alpha|=1 .\end{cases}\right.
$$

It is immediate from above that $\left\|\tilde{w}_{\alpha}\right\|\|\Phi(|\alpha|-1)\|<1$. Hence it follows from Proposition 3.2 (iii) that $T$ is a row contraction.

To see the if and only if part, first note that if $d=1$, then the notion of row contraction coincides with that of joint contraction. Suppose that $T$ is a joint contraction. Note that for $\alpha \in \mathbb{N}^{d} \backslash\{0\}$,

$$
\left\|w_{\alpha}\right\|^{2}=\frac{1}{4} \sum_{j=1}^{d} \frac{\alpha_{j}+1}{|\alpha|+1}=\frac{1}{4} \frac{|\alpha|+d}{|\alpha|+1} .
$$

It follows from Proposition 3.2 (ii) that for $\alpha=\varepsilon_{1}$,

$$
\left\|w_{\varepsilon_{1}}\right\|\|\Phi(1)\|=\frac{\sqrt{d+1}}{2 \sqrt{2}} 2 \phi(1)=\frac{\sqrt{d+1}}{\sqrt{2}} \frac{\sqrt{3}}{\sqrt{4}} \leqslant 1 .
$$

Thus $d \leqslant \frac{5}{3}<2$. Since $d$ is a positive integer, we conclude that $d$ must be 1 . This completes the proof of aforementioned assertions.

REMARK 3.4. The operator-valued multishift discussed in the foregoing example may be realized as a $d$-tuple of operators of multiplication by the coordinate functions on a reproducing kernel Hilbert space $\mathcal{H}(\kappa)$ of $\mathbb{C}^{2}$-valued holomorphic functions on the open unit ball $\mathbb{B}^{d}$ in $\mathbb{C}^{d}$. Indeed, the positive definite kernel $\kappa$ is given by

$$
\kappa(z, w)=\left(\begin{array}{cc}
\frac{1}{(1-\langle z, w\rangle)^{2}} & \frac{\langle z, w\rangle}{(1-\langle z, w\rangle)} \\
\frac{\langle z, w\rangle}{(1-\langle z, w\rangle)} & \frac{1}{(1-\langle z, w\rangle)^{2}}
\end{array}\right)=\left(\begin{array}{cc}
1 & 0 \\
0 & 1
\end{array}\right)+\sum_{\substack{\alpha \in \mathbb{N}^{d} \\
|\alpha| \geq 1}} \frac{|\alpha| !}{\alpha !}\left(\begin{array}{cc}
|\alpha|+1 & 1 \\
1 & |\alpha|+1
\end{array}\right) z^{\alpha} \bar{w}^{\alpha}
$$

for all $z$ and $w$ in $\mathbb{B}^{d}$. To see this, first note that if $\kappa_{1}$ and $\kappa_{2}$ are two positive definite kernels such that $\kappa_{2} \leq \kappa_{1}$, then $\kappa$ given by

$$
\kappa:=\left(\begin{array}{cc}
\kappa_{1} & \kappa_{2} \\
\kappa_{2} & \kappa_{1}
\end{array}\right)
$$


is a positive definite kernel. Now consider the positive definite kernels $\kappa_{1}$ and $\kappa_{2}$ given by

$$
\kappa_{1}(z, w)=\frac{1}{(1-\langle z, w\rangle)^{2}} \text { and } \kappa_{2}(z, w)=\frac{\langle z, w\rangle}{(1-\langle z, w\rangle)} \text { for all } z, w \in \mathbb{B}^{d} .
$$

Then for all $z, w \in \mathbb{B}^{d}$,

$$
\kappa(z, w)=\left(\begin{array}{cc}
\frac{1}{(1-\langle z, w\rangle)^{2}} & \frac{\langle z, w\rangle}{(1-\langle z, w\rangle)} \\
\frac{\langle z, w\rangle}{(1-\langle z, w\rangle)} & \frac{1}{(1-\langle z, w\rangle)^{2}}
\end{array}\right)=\left(\begin{array}{cc}
1 & 0 \\
0 & 1
\end{array}\right)+\sum_{\substack{\alpha \in \mathbb{N}^{d} \\
|\alpha| \geq 1}} \frac{|\alpha| !}{\alpha !}\left(\begin{array}{cc}
|\alpha|+1 & 1 \\
1 & |\alpha|+1
\end{array}\right) z^{\alpha} \bar{w}^{\alpha}
$$

is a positive definite kernel. Consider the multisequence $\mathscr{B}=\left\{B_{\alpha} \in M_{2}(\mathbb{C}): \alpha \in\right.$ $\mathbb{N}^{d}$ \} given by

$$
B_{\alpha}= \begin{cases}\left(\begin{array}{ll}
1 & 0 \\
0 & 1
\end{array}\right) & \text { if } \alpha=0 \\
\sqrt{\frac{|\alpha| !}{\alpha !}}\left(\begin{array}{cc}
|\alpha|+1 & 1 \\
1 & |\alpha|+1
\end{array}\right)^{1 / 2} & \text { if }|\alpha| \geq 1\end{cases}
$$

Then we get

$$
B_{\alpha}= \begin{cases}\left(\begin{array}{ll}
1 & 0 \\
0 & 1
\end{array}\right) & \text { if } \alpha=0, \\
\frac{1}{2} \sqrt{\frac{|\alpha| !}{\alpha !}}\left(\begin{array}{ll}
\sqrt{|\alpha|+2}+\sqrt{|\alpha|} & \sqrt{|\alpha|+2}-\sqrt{|\alpha|} \\
\sqrt{|\alpha|+2}-\sqrt{|\alpha|} & \sqrt{|\alpha|+2}+\sqrt{|\alpha|}
\end{array}\right) & \text { if }|\alpha| \geqslant 1 .\end{cases}
$$

Thus the $d$-tuple of operators of multiplication by the coordinate functions on the reproducing kernel Hilbert space $\mathcal{H}(\kappa)$ associated with positive definite kernel $\kappa$ may be realized as the commuting operator-valued multishift with operator weights $\left\{A_{\alpha}^{(j)}=B_{\alpha} B_{\alpha+\varepsilon_{j}}^{-1}: \alpha \in \mathbb{N}^{d}, j=1, \ldots, d\right\}$ (see Remark 5.2). Hence,

$$
\begin{aligned}
& A_{\alpha}^{(j)}=\frac{1}{2} \sqrt{\frac{\alpha_{j}+1}{|\alpha|+1}}\left(\begin{array}{ll}
\sqrt{\frac{|\alpha|+2}{|\alpha|+3}}+\sqrt{\frac{|\alpha|}{|\alpha|+1}} & \sqrt{\frac{|\alpha|+2}{|\alpha|+3}}-\sqrt{\frac{|\alpha|}{|\alpha|+1}} \\
\sqrt{\frac{|\alpha|+2}{|\alpha|+3}}-\sqrt{\frac{|\alpha|}{|\alpha|+1}} & \sqrt{\frac{|\alpha|+2}{|\alpha|+3}}+\sqrt{\frac{|\alpha|}{|\alpha|+1}}
\end{array}\right), \quad \alpha \in \mathbb{N}^{d} \backslash\{0\}, \\
& A_{0}^{(j)}=\frac{1}{2 \sqrt{3}}\left(\begin{array}{cc}
\sqrt{3}+1 & 1-\sqrt{3} \\
1-\sqrt{3} & \sqrt{3}+1
\end{array}\right) \text {. }
\end{aligned}
$$

As in [5, Sections 5.3, 5.4], the joint subnormal, joint hyponormal and joint $m$-isometric operator-valued multishifts can be characterized analogously.

\section{Strong Circularity, Analyticity and Wandering Subspace Property}

A commuting $d$-tuple $S=\left(S_{1}, \ldots, S_{d}\right)$ on a Hilbert space $\mathcal{H}$ is said to be circular if for every $\lambda=\left(\lambda_{1}, \ldots, \lambda_{d}\right) \in \mathbb{T}^{d}$, there exists a unitary operator $U_{\lambda}$ on $\mathcal{H}$ such that

$$
U_{\lambda}^{*} S_{j} U_{\lambda}=\lambda_{j} S_{j} \text { for all } j=1, \ldots, d .
$$

Further, $S$ is said to be strongly circular if $U_{\lambda}$ can be chosen to be a strongly continuous unitary representation of $\mathbb{T}^{d}$ in the following sense: For every $h \in \mathcal{H}$, the function $\lambda \mapsto U_{\lambda} h$ is continuous on $\mathbb{T}^{d}$.

The above notion for $d=1$ was introduced and studied in [1. Since then these operators became a centre of attraction and were studied considerably thereafter (refer to [32, 16, 29], 2]). The circularity of a classical multishift was first obtained in [24, Corollary 3]. The circularity of a weighted shift on a directed tree 
was established in [23, Theorem 3.3.1]. In view of Proposition 2.6, the following result generalizes [5, Proposition 3.2.1] and a special case of [23, Theorem 3.3.1].

Proposition 4.1. Let $T=\left(T_{1}, \ldots, T_{d}\right)$ be a commuting operator-valued multishift on $\mathcal{H}=\oplus_{\alpha \in \mathbb{N}^{d}} H_{\alpha}$ with operator weights $\left\{A_{\alpha}^{(j)}: \alpha \in \mathbb{N}^{d}, j=1, \ldots, d\right\}$. Then $T$ is strongly circular.

Proof. For $\lambda=\left(\lambda_{1}, \ldots, \lambda_{d}\right) \in \mathbb{T}^{d}$, define the linear operator $U_{\lambda}$ on $\mathcal{H}$ by

$$
U_{\lambda}\left(\oplus_{\alpha \in \mathbb{N}^{d}} x_{\alpha}\right)=\oplus_{\alpha \in \mathbb{N}^{d}} \bar{\lambda}^{\alpha} x_{\alpha}, \quad \oplus_{\alpha \in \mathbb{N}^{d}} x_{\alpha} \in \mathcal{H} .
$$

Clearly, $U_{\lambda}$ is a unitary operator on $\mathcal{H}$ and its adjoint is given by

$$
U_{\lambda}^{*}\left(\oplus_{\alpha \in \mathbb{N}^{d}} x_{\alpha}\right)=\oplus_{\alpha \in \mathbb{N}^{d}} \lambda^{\alpha} x_{\alpha}, \quad \oplus_{\alpha \in \mathbb{N}^{d}} x_{\alpha} \in \mathcal{H} .
$$

For $\oplus_{\alpha \in \mathbb{N}^{d}} x_{\alpha} \in \mathcal{H}$ and $j \in\{1, \ldots, d\}$, we have

$$
\begin{aligned}
U_{\lambda}^{*} T_{j} U_{\lambda}\left(\oplus_{\alpha \in \mathbb{N}^{d}} x_{\alpha}\right) & =U_{\lambda}^{*} T_{j}\left(\oplus_{\alpha \in \mathbb{N}^{d}} \bar{\lambda}^{\alpha} x_{\alpha}\right)=U_{\lambda}^{*}\left(\oplus_{\alpha \in \mathbb{N}^{d}} \bar{\lambda}^{\alpha-\varepsilon_{j}} A_{\alpha-\varepsilon_{j}}^{(j)} x_{\alpha-\varepsilon_{j}}\right) \\
& =\oplus_{\alpha \in \mathbb{N}^{d}} \lambda^{\alpha} \bar{\lambda}^{\alpha-\varepsilon_{j}} A_{\alpha-\varepsilon_{j}}^{(j)} x_{\alpha-\varepsilon_{j}}=\oplus_{\alpha \in \mathbb{N}^{d}} \lambda_{j} A_{\alpha-\varepsilon_{j}}^{(j)} x_{\alpha-\varepsilon_{j}} \\
& =\lambda_{j} T_{j}\left(\oplus_{\alpha \in \mathbb{N}^{d}} x_{\alpha}\right) .
\end{aligned}
$$

This proves that $T$ is circular. To see the strong circularity, let $\left\{\lambda^{(n)}\right\}_{n \in \mathbb{N}}$ be a sequence in $\mathbb{T}^{d}$ which converges to $\lambda \in \mathbb{T}^{d}$. Then for $\oplus_{\alpha \in \mathbb{N}^{d}} x_{\alpha} \in \mathcal{H}$, we get

$$
\left\|\left(U_{\lambda^{(n)}}-U_{\lambda}\right) \oplus_{\alpha \in \mathbb{N}^{d}} x_{\alpha}\right\|^{2}=\sum_{\alpha \in \mathbb{N}^{d}}\left|{\overline{\lambda^{(n)}}}^{\alpha}-\bar{\lambda}^{\alpha}\right|^{2}\left\|x_{\alpha}\right\|^{2} .
$$

Let $\epsilon>0$. Then there exists $n_{1} \in \mathbb{N}$ such that $\sum_{\substack{\alpha \in \mathbb{N}^{d} \\|\alpha|>n_{1}}}\left\|x_{\alpha}\right\|^{2}<\epsilon$. Further, for each $\alpha \in \mathbb{N}^{d}$, there exists $n_{\alpha} \in \mathbb{N}$ such that $\left|{\overline{\lambda^{(n)}}}^{\alpha}-\bar{\lambda}^{\alpha}\right|^{2}<\epsilon$ for all $n \geqslant n_{\alpha}$. Let $n_{2}=\max \left\{n_{\alpha}:|\alpha| \leqslant n_{1}\right\}$. Then for all $n \geqslant n_{2}$, we have

$$
\begin{aligned}
\left\|\left(U_{\lambda^{(n)}}-U_{\lambda}\right) \oplus_{\alpha \in \mathbb{N}^{d}} x_{\alpha}\right\|^{2} & =\sum_{\substack{\alpha \in \mathbb{N}^{d}\\
}}\left|{\overline{\lambda^{(n)}}}^{\alpha}-\bar{\lambda}^{\alpha}\right|^{2}\left\|x_{\alpha}\right\|^{2} \\
& <\sum_{\substack{\alpha \in \mathbb{N}^{d} \\
|\alpha| \leqslant n_{1}}}\left|\bar{\lambda}^{\alpha}-\bar{\lambda}^{\alpha}\right|^{2}\left\|x_{\alpha}\right\|^{2}+\sum_{\substack{\alpha \in \mathbb{N}^{d} \\
|\alpha|>n_{1}}}\left|\overline{\lambda^{(n)}}{ }^{\alpha}-\bar{\lambda}^{\alpha}\right|^{2}\left\|x_{\alpha}\right\|^{2} \\
& <\sum_{\substack{\alpha \in \mathbb{N}^{d} \\
|\alpha| \leqslant n_{1}}}\left\|x_{\alpha}\right\|^{2}+4 \epsilon .
\end{aligned}
$$

This completes the proof.

The following result is an immediate consequence of the preceding proposition.

Corollary 4.2. Let $T=\left(T_{1}, \ldots, T_{d}\right)$ be a commuting operator-valued multishift on $\mathcal{H}=\oplus_{\alpha \in \mathbb{N}^{d}} H_{\alpha}$ with operator weights $\left\{A_{\alpha}^{(j)}: \alpha \in \mathbb{N}^{d}, j=1, \ldots, d\right\}$. Then $\sigma(T), \sigma_{p}(T), \sigma_{l}(T)$ and $\sigma_{e}(T)$ have the polycircular symmetry. In particular, $\sigma(T)$ coincides with $\sigma\left(T^{*}\right)$.

The proof of the following proposition goes essentially along the lines of the proof of [5, Proposition 3.2.4]. The ideas involved in this proof are similar to that of [8. Lemma 3.8]. However, we include all the details for the sake of completeness.

Proposition 4.3. Let $T=\left(T_{1}, \ldots, T_{d}\right)$ be a commuting operator-valued multishift on $\mathcal{H}=\oplus_{\alpha \in \mathbb{N}^{d}} H_{\alpha}$ with operator weights $\left\{A_{\alpha}^{(j)}: \alpha \in \mathbb{N}^{d}, j=1, \ldots, d\right\}$. Then the Taylor spectrum of $T$ is connected. 
Proof. In view of Corollary 4.2, we prove that $\sigma\left(T^{*}\right)$ is connected. By Remark 2.5. $0 \in \sigma\left(T^{*}\right)$. Let $F_{1}$ be the connected component of $\sigma\left(T^{*}\right)$ containing 0 and let $F_{2}=\sigma\left(T^{*}\right) \backslash F_{1}$. By the Shilov Idempotent Theorem [10, Application 5.24], there exist closed invariant subspaces $\mathcal{W}_{1}, \mathcal{W}_{2}$ of $T^{*}$ such that $\mathcal{H}=\mathcal{W}_{1}+\mathcal{W}_{2}$ (vector space direct sum of $\mathcal{W}_{1}$ and $\left.\mathcal{W}_{2}\right)$ and $\sigma\left(T^{*} \mid \mathcal{W}_{l}\right)=F_{l}$ for $l=1,2$. For $k \in \mathbb{N}$, let $D^{(k)}=\left(T_{1}^{* k}, \ldots, T_{d}^{* k}\right)$ and $h \in \operatorname{ker} D^{(k)}$. Then $h=x+y$ for $x \in \mathcal{W}_{1}$ and $y \in \mathcal{W}_{2}$. It follows that $T_{j}^{* k} x=0=T_{j}^{* k} y$ for all $j=1, \ldots, d$. If $y$ is nonzero, then $0 \in \sigma_{p}\left(\left.D^{(k)}\right|_{\mathcal{W}_{2}}\right) \subseteq \sigma\left(\left.D^{(k)}\right|_{\mathcal{W}_{2}}\right)$, and hence by the spectral mapping property [10], $0 \in \sigma\left(D^{(1)} \mid \mathcal{W}_{2}\right)=\sigma\left(T^{*} \mid \mathcal{W}_{2}\right)$. Since $0 \notin F_{2}$, we must have $y=0$. It follows that $\mathcal{W}_{1}$ contains the subspace $\cup_{k \in \mathbb{N}} \operatorname{ker} D^{(k)}$, which is dense in $\mathcal{H}$ by Corollary 2.4] Hence $\mathcal{W}_{1}=\mathcal{H}$. Thus the Taylor spectrum of $T^{*}$ is equal to $F_{1}$ and hence $\sigma\left(T^{*}\right)$ is connected.

REMARK 4.4. As a consequence of Corollary 4.2 and the preceding proposition, it follows that if $T=\left(T_{1}, \ldots, T_{d}\right)$ is a commuting operator-valued multishift on $\mathcal{H}=\oplus_{\alpha \in \mathbb{N}^{d}} H_{\alpha}$ with operator weights $\left\{A_{\alpha}^{(j)}: \alpha \in \mathbb{N}^{d}, j=1, \ldots, d\right\}$, then the Taylor spectrum of $T$ is Reinhardt.

THEOREM 4.5. Let $T=\left(T_{1}, \ldots, T_{d}\right)$ be a commuting operator-valued multishift on $\mathcal{H}=\oplus_{\alpha \in \mathbb{N}^{d}} H_{\alpha}$ with operator weights $\left\{A_{\alpha}^{(j)}: \alpha \in \mathbb{N}^{d}, j=1, \ldots, d\right\}$. Then the following statements hold:

(i) $T$ is separately analytic. That is, for each $j \in\{1, \ldots, d\}, T_{j}$ is analytic.

(ii) $T$ has wandering subspace property. That is,

$$
\bigvee_{\alpha \in \mathbb{N}^{d}} T^{\alpha}\left(\operatorname{ker} T^{*}\right)=\mathcal{H}
$$

Proof. Let $j \in\{1, \ldots, d\}$. For each $k \geqslant 1$, consider the subspace $M_{k}^{(j)}$ of $\mathcal{H}$ given by

$$
M_{k}^{(j)}=\left\{\oplus_{\alpha \in \mathbb{N}^{d}} x_{\alpha}: x_{\alpha}=0 \text { for } \alpha_{j} \leqslant k-1\right\}
$$

and note that $\operatorname{ran} T_{j}^{k} \subseteq M_{k}^{(j)}$. Hence

$$
\bigcap_{k \geqslant 1} \operatorname{ran} T_{j}^{k} \subseteq \bigcap_{k \geqslant 1} M_{k}^{(j)}
$$

Now suppose that $x=\oplus_{\alpha \in \mathbb{N}^{d}} x_{\alpha} \in \bigcap_{k \geqslant 1} M_{k}^{(j)}$. Then for every $\alpha \in \mathbb{N}^{d}, x_{\alpha}=0$ as there is a $k \in \mathbb{N}$ bigger than $\alpha_{j}$ such that $x \in M_{k}^{(j)}$. Hence $x=0$. Thus

$$
\bigcap_{k \geqslant 1} \operatorname{ran} T_{j}^{k} \subseteq \bigcap_{k \geqslant 1} M_{k}^{(j)}=\{0\} .
$$

This completes the proof of (i).

To see (ii), let $\mathcal{K}=\bigvee_{\alpha \in \mathbb{N}^{d}} T^{\alpha}\left(\operatorname{ker} T^{*}\right)$. To show $\mathcal{K}=\mathcal{H}$, it is sufficient to show that $M_{\alpha} \subseteq \mathcal{K}$ for every $\alpha \in \mathbb{N}^{d}$, where

$$
M_{\alpha}=\left\{\oplus_{\beta \in \mathbb{N}^{d}} x_{\beta} \in \mathcal{H}: x_{\beta}=0 \text { if } \beta \neq \alpha\right\} .
$$

We prove by mathematical induction on $k \in \mathbb{N}$ that if $|\alpha|=k$, then $M_{\alpha} \subseteq \mathcal{K}$. Note that $\operatorname{ker} T^{*} \subseteq \mathcal{K}$ and hence it follows from (3) that $M_{0} \subseteq \mathcal{K}$. Thus the induction hypothesis holds for $k=0$. Suppose that it is true for some $k \in \mathbb{N}$. Let $\alpha \in \mathbb{N}^{d}$ be such that $|\alpha|=k+1$. Then by induction hypothesis $M_{\alpha-\varepsilon_{j}} \subseteq \mathcal{K}$ for all $j \in\{1, \ldots, d\}$. Since $\mathcal{K}$ is closed and $T_{j}$-invariant, it follows that

$$
\overline{T_{j} M_{\alpha-\varepsilon_{j}}}=\left\{\oplus_{\beta \in \mathbb{N}^{d}} x_{\beta}: x_{\beta}=0 \text { if } \beta \neq \alpha \text { and } x_{\alpha} \in \overline{\operatorname{ran} A_{\alpha-\varepsilon_{j}}^{(j)}}\right\} \subseteq \mathcal{K} .
$$


Thus

$$
\left\{\oplus_{\beta \in \mathbb{N}^{d}} x_{\beta}: x_{\beta}=0 \text { if } \beta \neq \alpha \text { and } x_{\alpha} \in \sum_{j=1}^{d} \overline{\operatorname{ran} A_{\alpha-\varepsilon_{j}}^{(j)}}\right\} \subseteq \mathcal{K} .
$$

Further, from (3), it follows that

$$
\left\{\oplus_{\beta \in \mathbb{N}^{d}} x_{\beta}: x_{\beta}=0 \text { if } \beta \neq \alpha \text { and } x_{\alpha} \in \bigcap_{j=1}^{d} \operatorname{ker} A_{\alpha-\varepsilon_{j}}^{(j) *}\right\} \subseteq \operatorname{ker} T^{*} \subseteq \mathcal{K} .
$$

Combining (5) and (6), we get that $M_{\alpha} \subseteq \mathcal{K}$. This completes the proof.

Some immediate consequences of the preceding theorem are in order.

COROLlary 4.6. Let $T=\left(T_{1}, \ldots, T_{d}\right)$ be a commuting operator-valued multishift on $\mathcal{H}=\oplus_{\alpha \in \mathbb{N}^{d}} H_{\alpha}$ with operator weights $\left\{A_{\alpha}^{(j)}: \alpha \in \mathbb{N}^{d}, j=1, \ldots, d\right\}$. Then the following statements hold:

(i) $T$ is analytic.

(ii) The point spectrum $\sigma_{p}\left(T_{j}\right)$ of $T_{j}$ is contained in $\{0\}$ for each $j=1, \ldots, d$.

(iii) The joint point spectrum $\sigma_{p}(T)$ of $T$ is contained in $\{0\}$.

(iv) For each $j \in\{1, \ldots, d\}$,

$$
\bigvee_{k \in \mathbb{N}} \operatorname{ker} T_{j}^{* k}=\mathcal{H}=\bigvee_{\alpha \in \mathbb{N}^{d}} \operatorname{ker} T^{* \alpha}
$$

(v) For each $j \in\{1, \ldots, d\}$, the spectrum of $T_{j}$ is the closed disc of radius $r\left(T_{j}\right)$ centered at the origin, where $r(S)$ denotes the spectral radius of a bounded linear operator $S$.

Proof. The proof of (i) follows from the preceding proposition and the fact that $\bigcap_{\alpha \in \mathbb{N}^{d}} \operatorname{ran} T^{\alpha} \subseteq \bigcap_{k \in \mathbb{N}} \operatorname{ran} T_{j}^{k}$ for all $j=1, \ldots, d$.

To see (ii), fix $j \in\{1, \ldots, d\}$ and let $w \in \mathbb{C}$ be a non-zero eigenvalue of $T_{j}$. Then there exists a non-zero vector $x=\oplus_{\alpha \in \mathbb{N}^{d}} x_{\alpha} \in \mathcal{H}$ such that $T_{j} x=w x$. Hence $x \in \operatorname{ran} T_{j}^{k}$ for all $k \geqslant 1$. This contradicts the Theorem 4.5(i) and thus we conclude that $\sigma_{p}\left(T_{j}\right) \subseteq\{0\}$. The proof of (iii) follows from (ii).

Fix $j \in\{1, \ldots, d\}$. Then from Theorem 4.5)(i) and (i), it follows that

$$
\bigcap_{k \in \mathbb{N}} \operatorname{ran} T_{j}^{k}=\{0\}=\bigcap_{\alpha \in \mathbb{N}^{d}} \operatorname{ran} T^{\alpha}
$$

The desired conclusion now follows from taking the orthogonal complement on both sides of the above expression. This completes the verification of (iv).

Fix $j \in\{1, \ldots, d\}$. It follows from Proposition 4.1 that $\sigma\left(T_{j}\right)$ has circular symmetry. Further, proceeding along the lines of the proof of Proposition 4.3 and using (iv), one may deduce that $\sigma\left(T_{j}\right)$ is connected. Thus the spectrum of $T_{j}$ must be the closed disc of radius $r\left(T_{j}\right)$ centered at the origin. This verifies (v) and hence, completes the proof of the corollary.

From Theorem 4.5(ii) and Proposition 2.6. we get the following corollary which extends [5, Theorem 4.0.1] into the settings of directed Cartesian product of finitely many rooted directed trees not necessarily locally finite.

COROLlary 4.7. Let $\mathscr{T}=(V, \mathcal{E})$ be the directed Cartesian product of rooted directed trees $\mathscr{T}_{1}, \ldots, \mathscr{T}_{d}$ and let $S_{\boldsymbol{\lambda}}=\left(S_{1}, \ldots, S_{d}\right)$ be a commuting multishift on $\mathscr{T}$ with weights $\left\{\lambda_{v}^{(j)}: v \in V \backslash\{\right.$ root $\left.\}, j=1, \ldots, d\right\}$. Then $S_{\boldsymbol{\lambda}}$ has wandering subspace property. 
The following corollary gives a sufficient condition under which a commuting operator-valued multishift with non-invertible operator weights is unitarily equivalent to a commuting operator-valued multishift with invertible operator weights. It is an extended version of [5, Corollary 4.1.12] and its proof goes along the lines of that of [5, Corollary 4.1.12]. However, we include the details for the sake of completeness.

Corollary 4.8. Let $T=\left(T_{1}, \ldots, T_{d}\right)$ be a toral left invertible commuting operator-valued multishift on $\mathcal{H}=\oplus_{\alpha \in \mathbb{N}^{d}} H_{\alpha}$ with operator weights $\left\{A_{\alpha}^{(j)}: \alpha \in\right.$ $\left.\mathbb{N}^{d}, j=1, \ldots, d\right\}$. Suppose that $T^{\alpha}\left(\operatorname{ker} T^{*}\right)$ is orthogonal to $T^{\beta}\left(\operatorname{ker} T^{*}\right)$ whenever $\alpha \neq \beta, \alpha, \beta \in \mathbb{N}^{d}$. Then $T$ is unitarily equivalent to a commuting operator-valued multishift $\tilde{T}=\left(\tilde{T}_{1}, \ldots, \tilde{T}_{d}\right)$ on $\ell_{\mathrm{ker} T^{*}}^{2}\left(\mathbb{N}^{d}\right)$ with some invertible operator weights $\left\{\tilde{A}_{\alpha}^{(j)} \in \mathcal{B}\left(\operatorname{ker} T^{*}\right): \alpha \in \mathbb{N}^{d}, j=1, \ldots, d\right\}$.

Proof. As the spaces $T^{\alpha}\left(\operatorname{ker} T^{*}\right), \alpha \in \mathbb{N}^{d}$, are mutually orthogonal, it follows from Theorem 4.5)(ii) that $\mathcal{H}=\oplus_{\alpha \in \mathbb{N}^{d}} T^{\alpha}$ ( $\operatorname{ker} T^{*}$ ). Since $T$ is toral left invertible, it follows that for each $\alpha \in \mathbb{N}^{d}, \operatorname{dim} T^{\alpha}\left(\operatorname{ker} T^{*}\right)=\operatorname{dim} \operatorname{ker} T^{*}$ and $T^{\alpha}\left(\operatorname{ker} T^{*}\right)$ is a closed subspace of $\mathcal{H}$. Let $U_{\alpha}$ be a unitary from $T^{\alpha}\left(\operatorname{ker} T^{*}\right)$ onto $\operatorname{ker} T^{*}$. Now consider the operator $U: \mathcal{H} \rightarrow \ell_{\mathrm{ker} T^{*}}^{2}\left(\mathbb{N}^{d}\right)$ given by

$$
U\left(\oplus_{\alpha \in \mathbb{N}^{d}} x_{\alpha}\right)=\oplus_{\alpha \in \mathbb{N}^{d}} U_{\alpha} x_{\alpha}, \quad x_{\alpha} \in T^{\alpha}\left(\operatorname{ker} T^{*}\right) .
$$

Then it is easy to see that $U$ is a unitary operator. For each $\alpha \in \mathbb{N}^{d}$ and $j \in$ $\{1, \ldots, d\}$, let $\tilde{A}_{\alpha}^{(j)}:=\left.U_{\alpha+\varepsilon_{j}} T_{j}\right|_{T^{\alpha}\left(\operatorname{ker} T^{*}\right)} U_{\alpha}^{*}$. Clearly, each $\tilde{A}_{\alpha}^{(j)}$ is an invertible bounded linear operator on $\operatorname{ker} T^{*}$. Consider the operator-valued multishift $\tilde{T}=$ $\left(\tilde{T}_{1}, \ldots, \tilde{T}_{d}\right)$ on $\ell_{\mathrm{ker} T^{*}}^{2}\left(\mathbb{N}^{d}\right)$ with operator weights $\left\{\tilde{A}_{\alpha}^{(j)}: \alpha \in \mathbb{N}^{d}, j=1, \ldots, d\right\}$. Then for $\oplus_{\alpha \in \mathbb{N}^{d}} x_{\alpha} \in \mathcal{H}, x_{\alpha} \in T^{\alpha}\left(\operatorname{ker} T^{*}\right)$ and $j=1, \ldots, d$, we get

$$
\begin{aligned}
U T_{j}\left(\oplus_{\alpha \in \mathbb{N}^{d}} x_{\alpha}\right) & =U\left(\oplus_{\alpha \in \mathbb{N}^{d}} T_{j} x_{\alpha}\right)=U\left(\left.\oplus_{\alpha \in \mathbb{N}^{d}} T_{j}\right|_{T^{\alpha}\left(\operatorname{ker} T^{*}\right)} x_{\alpha}\right) \\
& =U\left(\left.\oplus_{\alpha \in \mathbb{N}^{d}} T_{j}\right|_{T^{\alpha-\varepsilon_{j}}\left(\operatorname{ker} T^{*}\right)} x_{\alpha-\varepsilon_{j}}\right) \\
& =\left.\oplus_{\alpha \in \mathbb{N}^{d}} U_{\alpha} T_{j}\right|_{T^{\alpha-\varepsilon_{j}}\left(\operatorname{ker} T^{*}\right)} x_{\alpha-\varepsilon_{j}} \\
& =\oplus_{\alpha \in \mathbb{N}^{d}} \tilde{A}_{\alpha-\varepsilon_{j}}^{(j)} U_{\alpha-\varepsilon_{j}} x_{\alpha-\varepsilon_{j}}=\tilde{T}_{j} U\left(\oplus_{\alpha \in \mathbb{N}^{d}} x_{\alpha}\right) .
\end{aligned}
$$

This completes the proof.

\section{Operator-valued Multishifts with invertible operator weights}

This section is devoted to the study of function theory of operator-valued multishifts on $\ell_{H}^{2}\left(\mathbb{N}^{d}\right)$ with invertible operator weights, where $H$ is a complex separable HIlbert space. We begin with the observation that any such operator-valued multishift can be looked upon as a tuple of operators of multiplication by the coordinate functions on a Hilbert space of vector-valued formal power series. In this regard, we recall some preliminaries about the Hilbert space of vector-valued formal power series.

Let $H$ be a complex separable Hilbert space. By an $H$-valued formal power series we mean the series $\sum_{\alpha \in \mathbb{N}^{d}} x_{\alpha} z^{\alpha}, x_{\alpha} \in H$, without regard to convergence at any point $z \in \mathbb{C}^{d}$. Fix a multisequence $\mathscr{B}=\left\{W_{\alpha} \in \mathcal{G}(H): \alpha \in \mathbb{N}^{d}\right\}$. Then

$$
\mathcal{H}^{2}(\mathscr{B}):=\left\{\sum_{\alpha \in \mathbb{N}^{d}} x_{\alpha} z^{\alpha}: \sum_{\alpha \in \mathbb{N}^{d}}\left\|W_{\alpha} x_{\alpha}\right\|^{2}<\infty\right\}
$$

is a complex Hilbert space endowed with the following inner product: For $f(z)=$ $\sum_{\alpha \in \mathbb{N}^{d}} x_{\alpha} z^{\alpha}$ and $g(z)=\sum_{\alpha \in \mathbb{N}^{d}} y_{\alpha} z^{\alpha}$ in $\mathcal{H}^{2}(\mathscr{B})$,

$$
\langle f(z), g(z)\rangle_{\mathcal{H}^{2}(\mathscr{B})}:=\sum_{\alpha \in \mathbb{N}^{d}}\left\langle W_{\alpha} x_{\alpha}, W_{\alpha} y_{\alpha}\right\rangle_{H} .
$$


We refer to $\mathcal{H}^{2}(\mathscr{B})$ as a Hilbert space of $H$-valued formal power series. Note that $\left\|x z^{\alpha}\right\|_{\mathcal{H}^{2}(\mathscr{B})}=\left\|W_{\alpha} x\right\|_{H}$ for all $x \in H$ and $\alpha \in \mathbb{N}^{d}$.

Let $\mathscr{M}_{z}=\left(\mathscr{M}_{z_{1}}, \ldots, \mathscr{M}_{z_{d}}\right)$ denote the $d$-tuple of operators of multiplication by the coordinate functions $z_{j}$ defined on the subspace $\left\{\sum_{\substack{\alpha \in \mathbb{N}^{d} \\|\alpha| \leqslant n}} x_{\alpha} z^{\alpha}: x_{\alpha} \in H, n \in \mathbb{N}\right\}$ of polynomials in $\mathcal{H}^{2}(\mathscr{B})$ by

$$
\mathscr{M}_{z_{j}}\left(\sum_{\substack{\alpha \in \mathbb{N}^{d} \\|\alpha| \leqslant n}} x_{\alpha} z^{\alpha}\right)=\sum_{\substack{\alpha \in \mathbb{N}^{d} \\|\alpha| \leqslant n}} x_{\alpha} z^{\alpha+\varepsilon_{j}}, \quad j=1, \ldots, d .
$$

Thus $\mathscr{M}_{z_{j}}$ are densely defined linear operators on $\mathcal{H}^{2}(\mathscr{B})$ which may not be bounded in general. The following proposition shows that an operator-valued multishift with invertible operator weights can be recognized as the $d$-tuple of operators of multiplication by the coordinate functions on some Hilbert space of formal power series.

THEOREM 5.1. Let $H$ be a complex separable Hilbert space and $T=\left(T_{1}, \ldots, T_{d}\right)$ be a commuting operator-valued multishift on $\ell_{H}^{2}\left(\mathbb{N}^{d}\right)$ with invertible operator weights $\left\{A_{\alpha}^{(j)}: \alpha \in \mathbb{N}^{d}, j=1, \ldots, d\right\}$. Let $\mathscr{B}=\left\{B_{\alpha} \in \mathcal{G}(H): \alpha \in \mathbb{N}^{d}\right\}$, where $B_{\alpha}$ is as defined in Proposition 2.3 (see note after the statement of Proposition [2.3). Then for each $j \in\{1, \ldots, d\}$, the operator $\mathscr{M}_{z_{j}}$ of multiplication by the coordinate function $z_{j}$ on $\mathcal{H}^{2}(\mathscr{B})$ is bounded. Further, there exists a unitary $U: \ell_{H}^{2}\left(\mathbb{N}^{d}\right) \rightarrow \mathcal{H}^{2}(\mathscr{B})$ such that $\mathscr{M}_{z_{j}} U=U T_{j}$ for all $j=1, \ldots, d$. Moreover, the following statements hold:

(i) For all $\sum_{\alpha \in \mathbb{N}^{d}} x_{\alpha} z^{\alpha} \in \mathcal{H}^{2}(\mathscr{B})$,

$$
\mathscr{M}_{z_{j}}^{*}\left(\sum_{\alpha \in \mathbb{N}^{d}} x_{\alpha} z^{\alpha}\right)=\sum_{\alpha \in \mathbb{N}^{d}} B_{\alpha}^{-1} A_{\alpha}^{(j) *} B_{\alpha+\varepsilon_{j}} x_{\alpha+\varepsilon_{j}} z^{\alpha} .
$$

(ii) $A$ point $w \in \sigma_{p}\left(\mathscr{M}_{z}^{*}\right)$ if and only if there exists a non-zero vector $x$ in $H$ such that $\sum_{\alpha \in \mathbb{N}^{d}}\left|w^{\alpha}\right|^{2}\left\|B_{\alpha}^{*-1} x\right\|^{2}<\infty$.

Proof. It follows easily from (11) and Proposition 2.3)(ii) that $\mathscr{M}_{z_{j}}$ is bounded on $\mathcal{H}^{2}(\mathscr{B})$. For each $\alpha \in \mathbb{N}^{d}$ and $j \in\{1, \ldots, d\}, A_{\alpha}^{(j)}$ is invertible so is $B_{\alpha}$. Thus $B_{\alpha} H=H$ and $\ell_{H}^{2}\left(\mathbb{N}^{d}\right)=\oplus_{\alpha \in \mathbb{N}^{d}} B_{\alpha} H$. It follows that for each $x \in \ell_{H}^{2}\left(\mathbb{N}^{d}\right)$, there exists a unique sequence $\left\{x_{\alpha}\right\}_{\alpha \in \mathbb{N}^{d}}$ in $H$ such that $x=\oplus_{\alpha \in \mathbb{N}^{d}} B_{\alpha} x_{\alpha}$. Define the $\operatorname{map} U: \ell_{H}^{2}\left(\mathbb{N}^{d}\right) \rightarrow \mathcal{H}^{2}(\mathscr{B})$ by

$$
U\left(\oplus_{\alpha \in \mathbb{N}^{d}} B_{\alpha} x_{\alpha}\right)=\sum_{\alpha \in \mathbb{N}^{d}} x_{\alpha} z^{\alpha}
$$

It is easy to verify that $U$ is a surjective isometry and hence a unitary. Fix $j \in$ $\{1, \ldots, d\}$. Then for $\oplus_{\alpha \in \mathbb{N}^{d}} B_{\alpha} x_{\alpha} \in \ell_{H}^{2}\left(\mathbb{N}^{d}\right)$ and using the Proposition 2.3(ii), we have

$$
\begin{aligned}
\mathscr{M}_{z_{j}} U\left(\oplus_{\alpha \in \mathbb{N}^{d}} B_{\alpha} x_{\alpha}\right) & =\mathscr{M}_{z_{j}}\left(\sum_{\alpha \in \mathbb{N}^{d}} x_{\alpha} z^{\alpha}\right)=\sum_{\alpha \in \mathbb{N}^{d}} x_{\alpha} z^{\alpha+\varepsilon_{j}} \\
& =\sum_{\alpha \in \mathbb{N}^{d}} x_{\alpha-\varepsilon_{j}} z^{\alpha}=U\left(\oplus_{\alpha \in \mathbb{N}^{d}} B_{\alpha} x_{\alpha-\varepsilon_{j}}\right) \\
& =U\left(\oplus_{\alpha \in \mathbb{N}^{d}} A_{\alpha-\varepsilon_{j}}^{(j)} B_{\alpha-\varepsilon_{j}} x_{\alpha-\varepsilon_{j}}\right) \\
& =U T_{j}\left(\oplus_{\alpha \in \mathbb{N}^{d}} B_{\alpha} x_{\alpha}\right) .
\end{aligned}
$$


This completes the proof of the first half of the proposition. For the moreover part, let $\sum_{\alpha \in \mathbb{N}^{d}} x_{\alpha} z^{\alpha} \in \mathcal{H}^{2}(\mathscr{B})$ and note that

$$
\begin{aligned}
\mathscr{M}_{z_{j}}^{*}\left(\sum_{\alpha \in \mathbb{N}^{d}} x_{\alpha} z^{\alpha}\right) & =U T_{j}^{*} U^{*}\left(\sum_{\alpha \in \mathbb{N}^{d}} x_{\alpha} z^{\alpha}\right)=U T_{j}^{*}\left(\oplus_{\alpha \in \mathbb{N}^{d}} B_{\alpha} x_{\alpha}\right) \\
& =U\left(\oplus_{\alpha \in \mathbb{N}^{d}} A_{\alpha}^{(j) *} B_{\alpha+\varepsilon_{j}} x_{\alpha+\varepsilon_{j}}\right) \\
& =U\left(\oplus_{\alpha \in \mathbb{N}^{d}} B_{\alpha} B_{\alpha}^{-1} A_{\alpha}^{(j) *} B_{\alpha+\varepsilon_{j}} x_{\alpha+\varepsilon_{j}}\right) \\
& =\sum_{\alpha \in \mathbb{N}^{d}} B_{\alpha}^{-1} A_{\alpha}^{(j) *} B_{\alpha+\varepsilon_{j}} x_{\alpha+\varepsilon_{j}} z^{\alpha} .
\end{aligned}
$$

This establishes (i).

To see (ii), let $w \in \sigma_{p}\left(\mathscr{M}_{z}^{*}\right)$ and $f_{w}(z)=\sum_{\alpha \in \mathbb{N}^{d}} x_{\alpha} z^{\alpha} \in \mathcal{H}^{2}(\mathscr{B})$ be an eigenvector corresponding to $w$. Then

$$
\mathscr{M}_{z_{j}}^{*} f_{w}(z)=w_{j} f_{w}(z) \text { for all } j=1, \ldots, d .
$$

Using (i) and comparing the coefficients of $z^{\alpha}$ on both sides of (17), we get

$$
B_{\alpha}^{-1} A_{\alpha}^{(j) *} B_{\alpha+\varepsilon_{j}} x_{\alpha+\varepsilon_{j}}=w_{j} x_{\alpha} \text { for all } \alpha \in \mathbb{N}^{d} \text { and } j=1, \ldots, d .
$$

Consequently, for all $\alpha \in \mathbb{N}^{d}$ and $j \in\{1, \ldots, d\}$,

$$
x_{\alpha+\varepsilon_{j}}=w_{j} B_{\alpha+\varepsilon_{j}}^{-1} A_{\alpha}^{(j) *-1} B_{\alpha} x_{\alpha} .
$$

It is easy to see that for fixed $x_{0} \in H, x_{\alpha}$ is well-defined for each $\alpha \in \mathbb{N}^{d}$. That is, $x_{\left(\alpha+\varepsilon_{j}\right)+\varepsilon_{k}}=x_{\left(\alpha+\varepsilon_{k}\right)+\varepsilon_{j}}$ for all $\alpha \in \mathbb{N}^{d}$ and $j, k=1, \ldots, d$. By repeated applications of (8) and using Proposition 2.3(ii), we get

$$
x_{\alpha}=w^{\alpha}\left(B_{\alpha}^{*} B_{\alpha}\right)^{-1} x_{0} \text { for all } \alpha \in \mathbb{N}^{d},
$$

where $x_{0}:=f_{w}(0)$. Thus $f_{w}(z)=\sum_{\alpha \in \mathbb{N}^{d}} w^{\alpha}\left(B_{\alpha}^{*} B_{\alpha}\right)^{-1} x_{0} z^{\alpha}$. As $f_{w} \in \mathcal{H}^{2}(\mathscr{B})$, we must have $\sum_{\alpha \in \mathbb{N}^{d}}\left|w^{\alpha}\right|^{2}\left\|B_{\alpha}^{*-1} x_{0}\right\|^{2}<\infty$.

Conversely, suppose that $\sum_{\alpha \in \mathbb{N}^{d}}\left|w^{\alpha}\right|^{2}\left\|B_{\alpha}^{*-1} x\right\|^{2}<\infty$ for some $w \in \mathbb{C}^{d}$ and a non-zero vector $x \in H$. Define

$$
g(z):=\sum_{\alpha \in \mathbb{N}^{d}} w^{\alpha}\left(B_{\alpha}^{*} B_{\alpha}\right)^{-1} x z^{\alpha} .
$$

Then $g$ is a non-zero element of $\mathcal{H}^{2}(\mathscr{B})$. From (i) and Proposition 2.3(ii), we get for all $j \in\{1, \ldots, d\}$,

$$
\begin{aligned}
\mathscr{M}_{z_{j}}^{*} g & =\sum_{\alpha \in \mathbb{N}^{d}} w^{\alpha+\varepsilon_{j}} B_{\alpha}^{-1} A_{\alpha}^{(j) *} B_{\alpha+\varepsilon_{j}}\left(B_{\alpha+\varepsilon_{j}}^{*} B_{\alpha+\varepsilon_{j}}\right)^{-1} x z^{\alpha} \\
& =w_{j} \sum_{\alpha \in \mathbb{N}^{d}} w^{\alpha} B_{\alpha}^{-1} B_{\alpha}^{*-1} x z^{\alpha}=w_{j} g .
\end{aligned}
$$

This completes the proof of the proposition.

REMARK 5.2. It can be easily verified that for a given multisequence $\left\{B_{\alpha} \in\right.$ $\left.\mathcal{G}(H): \alpha \in \mathbb{N}^{d}\right\}$ of invertible operators on $H$, if the $d$-tuple $\mathscr{M}_{z}=\left(\mathscr{M}_{z_{1}}, \ldots, \mathscr{M}_{z_{d}}\right)$ of operators of multiplication by the coordinate functions on $\mathcal{H}^{2}(\mathscr{B})$ is bounded, then it is unitarily equivalent to a commuting operator-valued multishift on $\ell_{H}^{2}\left(\mathbb{N}^{d}\right)$ with invertible operator weights $A_{\alpha}^{(j)}=B_{\alpha+\varepsilon_{j}} B_{\alpha}^{-1}, \alpha \in \mathbb{N}^{d}$ and $j=1, \ldots, d$. 
A natural question arises here is whether the Hilbert space $\mathcal{H}^{2}(\mathscr{B})$ obtained in the foregoing proposition can be realized as a reproducing kernel Hilbert space of holomorphic functions. Before proceeding towards an answer of this question, let us recall a definition from 24 . For $w \in \mathbb{C}^{d}$, let $E_{w}$ denote the linear map of evaluation at $w$ defined on the polynomials in $\mathcal{H}^{2}(\mathscr{B})$ onto $H$ by $E_{w} p=p(w)$. A point $w \in \mathbb{C}^{d}$ is called a bounded point evaluation (bpe) on $\mathcal{H}^{2}(\mathscr{B})$ if $E_{w}$ extends to a continuous linear map from $\mathcal{H}^{2}(\mathscr{B})$ onto $H$. By an abuse of notation, we denote the continuous extension of $E_{w}$ by $E_{w}$ itself.

In the following theorem, we determine the set of bounded point evaluations for a commuting operator-valued multishift with invertible operator weights.

Proposition 5.3. Let $H$ be a complex separable Hilbert space and let $T=$ $\left(T_{1}, \ldots, T_{d}\right)$ be a commuting operator-valued multishift on $\ell_{H}^{2}\left(\mathbb{N}^{d}\right)$ with invertible operator weights $\left\{A_{\alpha}^{(j)}: \alpha \in \mathbb{N}^{d}, j=1, \ldots, d\right\}$. Let $\mathscr{B}=\left\{B_{\alpha} \in \mathcal{G}(H): \alpha \in\right.$ $\left.\mathbb{N}^{d}\right\}$, where $B_{\alpha}$ is as defined in Proposition 2.3. Then the set of all bounded point evaluations on $\mathcal{H}^{2}(\mathscr{B})$ is contained in $\sigma_{p}\left(T^{*}\right)$ and is given by

$$
\Omega:=\left\{w \in \mathbb{C}^{d}: \sup _{x \in H,\|x\|=1} \sum_{\alpha \in \mathbb{N}^{d}}\left|w^{\alpha}\right|^{2}\left\|B_{\alpha}^{*-1} x\right\|^{2}<\infty\right\} .
$$

Proof. Let $w \in \mathbb{C}^{d}$ be a bounded point evaluation on $\mathcal{H}^{2}(\mathscr{B})$. Then the evaluation map $E_{w}: \mathcal{H}^{2}(\mathscr{B}) \rightarrow H$ is continuous. For $x \in H$, consider the vector $E_{w}^{*} x \in \mathcal{H}^{2}(\mathscr{B})$ and note that for any polynomial $p \in \mathcal{H}^{2}(\mathscr{B})$ and $j \in\{1, \ldots, d\}$,

$$
\begin{aligned}
\left\langle\mathscr{M}_{z_{j}}^{*} E_{w}^{*} x, p\right\rangle_{\mathcal{H}^{2}(\mathscr{B})} & =\left\langle E_{w}^{*} x, z_{j} p\right\rangle_{\mathcal{H}^{2}(\mathscr{B})}=\left\langle x, E_{w}\left(z_{j} p\right)\right\rangle_{H}=\left\langle x, w_{j} p(w)\right\rangle_{H} \\
& =\left\langle\bar{w}_{j} x, p(w)\right\rangle_{H}=\left\langle\bar{w}_{j} E_{w}^{*} x, p\right\rangle_{\mathcal{H}^{2}(\mathscr{B})} .
\end{aligned}
$$

Since the polynomials are dense in $\mathcal{H}^{2}(\mathscr{B})$, it follows that $\mathscr{M}_{z_{j}}^{*} E_{w}^{*} x=\bar{w}_{j} E_{w}^{*} x$ for all $j \in\{1, \ldots, d\}$. This shows that the set of all bounded point evaluations is contained in $\overline{\sigma_{p}\left(\mathscr{M}_{z}^{*}\right)}$ (the complex conjugate of $\sigma_{p}\left(\mathscr{M}_{z}^{*}\right)$ ). Since $\sigma_{p}\left(\mathscr{M}_{z}^{*}\right)$ has polycircular symmetry (see Corollary 4.2), it follows that the set of all bounded point evaluations is contained in $\sigma_{p}\left(\mathscr{M}_{z}^{*}\right)$. For the remaining part, we first claim that if $w \in \mathbb{C}^{d}$ is a bounded point evaluation, then

$$
f(z):=\sum_{\alpha \in \mathbb{N}^{d}} \bar{w}^{\alpha}\left(B_{\alpha}^{*} B_{\alpha}\right)^{-1} x z^{\alpha} \in \mathcal{H}^{2}(\mathscr{B}) .
$$

To see this, let $g(z)=\sum_{\alpha \in \mathbb{N}^{d}} x_{\alpha} z^{\alpha} \in \mathcal{H}^{2}(\mathscr{B})$. For $n \in \mathbb{N}$, set

$$
f_{n}(z):=\sum_{\substack{\alpha \in \mathbb{N}^{d} \\|\alpha| \leqslant n}} \bar{w}^{\alpha}\left(B_{\alpha}^{*} B_{\alpha}\right)^{-1} x z^{\alpha} \text { and } g_{n}(z):=\sum_{\substack{\alpha \in \mathbb{N}^{d} \\|\alpha| \leqslant n}} x_{\alpha} z^{\alpha} .
$$

Then for $n \in \mathbb{N}$, we get

$$
\begin{aligned}
\left\langle f_{n}(z), g(z)\right\rangle_{\mathcal{H}^{2}(\mathscr{B})} & =\left\langle\sum_{\substack{\alpha \in \mathbb{N}^{d} \\
|\alpha| \leqslant n}} \bar{w}^{\alpha}\left(B_{\alpha}^{*} B_{\alpha}\right)^{-1} x z^{\alpha}, \sum_{\alpha \in \mathbb{N}^{d}} x_{\alpha} z^{\alpha}\right\rangle_{\mathcal{H}^{2}(\mathscr{B})} \\
& =\sum_{\substack{\alpha \in \mathbb{N}^{d} \\
|\alpha| \leqslant n}}\left\langle\bar{w}^{\alpha} B_{\alpha}^{*-1} x, B_{\alpha} x_{\alpha}\right\rangle_{H}=\left\langle x, \sum_{\substack{\alpha \in \mathbb{N}^{d} \\
|\alpha| \leqslant n}} w^{\alpha} x_{\alpha}\right\rangle_{H}=\left\langle x, E_{w} g_{n}\right\rangle_{H} \\
& =\left\langle E_{w}^{*} x, g_{n}\right\rangle_{\mathcal{H}^{2}(\mathscr{B})} .
\end{aligned}
$$

This, in turn, implies that

$$
\left\|f_{n}\right\|_{\mathcal{H}^{2}(\mathscr{B})}=\sup _{\substack{g \in \mathcal{H}^{2}(\mathscr{B}) \\\|g\|=1}}\left|\left\langle f_{n}(z), g(z)\right\rangle_{\mathcal{H}^{2}(\mathscr{B})}\right| \leqslant\left\|E_{w}^{*} x\right\|_{\mathcal{H}^{2}(\mathscr{B})} \quad \text { for all } n \in \mathbb{N} \text {. }
$$


Thus $f \in \mathcal{H}^{2}(\mathscr{B})$ and hence, the claim stands verified. Further, taking $n \rightarrow \infty$ on both sides of (10), we get

$$
E_{w}^{*} x=\sum_{\alpha \in \mathbb{N}^{d}} \bar{w}^{\alpha}\left(B_{\alpha}^{*} B_{\alpha}\right)^{-1} x z^{\alpha} \text { for all } x \in H .
$$

Now the continuity of $E_{w}^{*}$ gives that

$$
\sup _{x \in H,\|x\|=1}\left\|E_{w}^{*} x\right\|^{2} \stackrel{\text { 111) }}{=} \sup _{x \in H,\|x\|=1} \sum_{\alpha \in \mathbb{N}^{d}}\left|w^{\alpha}\right|^{2}\left\|B_{\alpha}^{*-1} x\right\|^{2}<\infty .
$$

Thus the set of all bounded point evaluations is contained in $\Omega$.

Conversely, suppose that $w \in \Omega$. Then for each $x \in H$,

$$
g_{w, x}(z):=\sum_{\alpha \in \mathbb{N}^{d}} \bar{w}^{\alpha} B_{\alpha}^{-1} B_{\alpha}^{*-1} x z^{\alpha} \in \mathcal{H}^{2}(\mathscr{B}) .
$$

Now define $F_{w}: H \rightarrow \mathcal{H}^{2}(\mathscr{B})$ by $F_{w} x=g_{w, x}(z)$ for all $x \in H$. It follows from the definition of $\Omega$ that $F_{w}$ is a bounded linear map. Further, for any polynomial $p \in \mathcal{H}^{2}(\mathscr{B})$ and $x \in H$,

$$
\left\langle F_{w}^{*} p, x\right\rangle_{H}=\left\langle p, F_{w} x\right\rangle_{\mathcal{H}^{2}(\mathscr{B})}=\left\langle p, g_{w, x}(z)\right\rangle_{\mathcal{H}^{2}(\mathscr{B})}=\langle p(w), x\rangle_{H} .
$$

Thus $F_{w}^{*}=E_{w}$ and hence $w$ is a bounded point evaluation. This completes the proof of the proposition.

\section{REMARK 5.4.}

(i) An alternate verification of (11) can be seen as follows: Since 0 is always a bounded point evaluation, it is easy to see that $E_{0}^{*} x=x$ for all $x \in H$. Suppose that $w \in \mathbb{C}^{d}$ is a bounded point evaluation. Then for all $x, y \in H$, we get

$$
\left\langle E_{0} E_{w}^{*} x, y\right\rangle_{H}=\left\langle E_{w}^{*} x, E_{0}^{*} y\right\rangle_{\mathcal{H}^{2}(\mathscr{B})}=\langle x, y\rangle_{H} .
$$

This shows that $E_{0} E_{w}^{*} x=x$ for all $x \in H$. Since $E_{w}^{*} x$ is an eigenvector of $\mathscr{M}_{z}^{*}$ corresponding to eigenvalue $\bar{w}$, it follows from the proof of Theorem 5.1(ii) that there exists a non-zero vector $y \in H$ such that

$$
E_{w}^{*} x=\sum_{\alpha \in \mathbb{N}^{d}} \bar{w}^{\alpha}\left(B_{\alpha}^{*} B_{\alpha}\right)^{-1} y z^{\alpha} .
$$

Using $E_{0} E_{w}^{*} x=x$, we get $y=x$. This verifies (11).

(ii) Unlike the classical case (24, Proposition 19]), the inclusion in the preceding proposition may be strict in general. For example, consider the commuting operator-valued multishift $T=\left(T_{1}, \ldots, T_{d}\right)$ on $\ell_{\mathbb{C}^{2}}^{2}\left(\mathbb{N}^{d}\right)$ with operator weights given by $A_{\alpha}^{(j)}=\left(\begin{array}{cc}2 & 0 \\ 0 & \frac{1}{2}\end{array}\right)$ for all $\alpha \in \mathbb{N}^{d}$ and $j=1, \ldots, d$. Then it is easy to see that $B_{\alpha}=\left(\begin{array}{cc}2^{|\alpha|} & 0 \\ 0 & \frac{1}{2|\alpha|}\end{array}\right)$ for all $\alpha \in \mathbb{N}^{d}$. Let $e_{1}=(1,0)$ and $e_{2}=(0,1)$ be two orthonormal vectors in $\mathbb{C}^{2}$. Then observe that for $w=(1, \ldots, 1) \in \mathbb{C}^{d}$,

$$
\sum_{\alpha \in \mathbb{N}^{d}}\left|w^{\alpha}\right|^{2}\left\|B_{\alpha}^{*-1} e_{1}\right\|^{2}<\infty \text { but } \sum_{\alpha \in \mathbb{N}^{d}}\left|w^{\alpha}\right|^{2}\left\|B_{\alpha}^{*-1} e_{2}\right\|^{2}=\infty .
$$

Thus by Theorem [5.1(ii), $w \in \sigma_{p}\left(T^{*}\right)$ but $w$ is not a bounded point evaluation.

The observation in the above remark motivates us for the following proposition. 
Proposition 5.5. Let $n$ be a positive integer and $T=\left(T_{1}, \ldots, T_{d}\right)$ be a commuting operator-valued multishift on $\ell_{\mathbb{C}^{n}}^{2}\left(\mathbb{N}^{d}\right)$ with invertble operator weights given by

$$
A_{\alpha}^{(j)}:=\left(\begin{array}{cccc}
w_{1, \alpha}^{(j)} & 0 & \ldots & 0 \\
0 & w_{2, \alpha}^{(j)} & \ldots & 0 \\
\vdots & \vdots & \ddots & \vdots \\
0 & 0 & \ldots & w_{n, \alpha}^{(j)}
\end{array}\right)
$$

where $w_{k, \alpha}^{(j)} \in \mathbb{C}$ for all $\alpha \in \mathbb{N}^{d}, j=1, \ldots, d$ and $k=1, \ldots, n$. Let $\mathscr{B}=\left\{B_{\alpha} \in\right.$ $\left.\mathcal{G}(H): \alpha \in \mathbb{N}^{d}\right\}$, where $B_{\alpha}$ is as defined in Proposition 2.3. Let $\Omega$ be the set of all bounded point evaluations on $\mathcal{H}^{2}(\mathscr{B})$. Then the following statements hold:

(i) $T$ is unitarily equivalent to $W_{1} \oplus \cdots \oplus W_{n}$, where for each $k \in\{1, \ldots, n\}$, $W_{k}$ is the classical multishift on $\ell^{2}\left(\mathbb{N}^{d}\right)$ with weights $\left\{w_{k, \alpha}^{(j)}: \alpha \in \mathbb{N}^{d}, j=\right.$ $1, \ldots, d\}$.

(ii) $\mathcal{H}^{2}(\mathscr{B})=\mathcal{H}^{2}\left(\mathscr{B}_{1}\right) \oplus \cdots \oplus \mathcal{H}^{2}\left(\mathscr{B}_{n}\right)$, where for $k \in\{1, \ldots, n\}, \mathscr{B}_{k}=\left\{B_{k, \alpha}\right.$ : $\left.\alpha \in \mathbb{N}^{d}\right\}$ and $B_{k, \alpha}$ is given by the expression of $B_{\alpha}$ in Proposition 2.3 by replacing $A_{\alpha}^{(j)}$ with $w_{k, \alpha}^{(j)}$.

(iii) $\Omega=\cap_{k=1}^{n} \sigma_{p}\left(W_{k}^{*}\right)$.

Proof. Part (i) has already been established in [18, Proposition 3.4]. The conclusion in (ii) follows from the observations that $\ell_{\mathbb{C}^{n}}^{2}\left(\mathbb{N}^{d}\right)=\oplus_{k=1}^{n} \ell^{2}\left(\mathbb{N}^{d}\right)$ and $B_{\alpha}=\oplus_{k=1}^{n} B_{k, \alpha}$. To see (iii), suppose that $w \in \Omega$. Then by Theorem $[5.3$, we have

$$
\sup _{x \in \mathbb{C}^{n},\|x\|=1} \sum_{\alpha \in \mathbb{N}^{d}}\left|w^{\alpha}\right|^{2}\left\|B_{\alpha}^{*-1} x\right\|^{2}<\infty .
$$

Hence it follows from (ii) that

$$
\sum_{\alpha \in \mathbb{N}^{d}}\left|w^{\alpha}\right|^{2}\left|B_{k, \alpha}\right|^{-2}<\infty \text { for all } k=1, \ldots, n .
$$

Therefore, by Theorem 5.1(ii) (see also [24, pp. 220]), we get that $w \in \sigma_{p}\left(W_{k}^{*}\right)$ for all $k=1, \ldots, n$. This proves that $\Omega \subseteq \cap_{k=1}^{n} \sigma_{p}\left(W_{k}^{*}\right)$. The proof for the reverse inclusion is similar.

Let $f=\sum_{\alpha \in \mathbb{N}^{d}} x_{\alpha} z^{\alpha} \in \mathcal{H}^{2}(\mathscr{B})$ and $\left\{p_{n}\right\}_{n \in \mathbb{N}}$ be a sequence of polynomials converging to $f$ in $\mathcal{H}^{2}(\mathscr{B})$. Let $\Omega$ be the set of all bounded point evaluations on $\mathcal{H}^{2}(\mathscr{B})$. If $w \in \Omega$, then we define $f(w)$ by

$$
f(w):=E_{w} f=\lim _{n \rightarrow \infty} E_{w} p_{n}=\lim _{n \rightarrow \infty} p_{n}(w) .
$$

Since $f(w)$ is well-defined for all $w \in \Omega, f$ defines an $H$-valued function on $\Omega$. In other words, if $f \in \mathcal{H}^{2}(\mathscr{B})$, then $\left.f\right|_{\Omega}$ is an $H$-valued function on $\Omega$, where the restriction of $f$ on $\Omega$ should be interpreted in above sense. Consider the inner product space $\mathcal{H}(\kappa):=\left\{\left.f\right|_{\Omega}: f \in \mathcal{H}^{2}(\mathscr{B})\right\}$, with the inner product inherited from $\mathcal{H}^{2}(\mathscr{B})$. Then it is easy to see that $\mathcal{H}(\kappa)$ is a Hilbert space of $H$-valued functions on $\Omega$, and $H$-valued polynomials are dense in $\mathcal{H}(\kappa)$. Further, it follows from 31 , Chapter 6] that $\mathcal{H}(\kappa)$ is a reproducing kernel Hilbert space with the reproducing kernel $\kappa: \Omega \times \Omega \rightarrow \mathcal{B}(H)$ given by

$$
\kappa(z, w)=E_{z} E_{w}^{*} \stackrel{111}{=} \sum_{\alpha \in \mathbb{N}^{d}}\left(B_{\alpha}^{*} B_{\alpha}\right)^{-1} z^{\alpha} \bar{w}^{\alpha}, \quad z, w \in \Omega .
$$

It turns out that if $\Omega$ has non-empty interior, then the elements of $\mathcal{H}(\kappa)$ define $H$-valued holomorphic functions on the interior of $\Omega$. Indeed, we have the following proposition. 
Proposition 5.6. Let $\mathcal{H}(\kappa)$ be defined as above. If $\Omega$ has a non-empty interior, then $\mathcal{H}(\kappa)$ is a Hilbert space of $H$-valued holomorphic functions on the interior of $\Omega$.

Proof. Let $w=\left(w_{1}, \ldots, w_{d}\right)$ be any point in the interior of $\Omega$. Then there exists $\tilde{w}=\left(\tilde{w}_{1}, \ldots, \tilde{w}_{d}\right)$ in the interior of $\Omega$ such that $\left|w_{j}\right|<\left|\tilde{w}_{j}\right|$ for all $j=1, \ldots, d$. Let $\phi: \mathbb{N} \rightarrow \mathbb{N}^{d}$ be a bijective map and $f=\sum_{\alpha \in \mathbb{N}^{d}} x_{\alpha} z^{\alpha} \in \mathcal{H}(\kappa)$. Note that the sequence of polynomials $\left\{p_{n}\right\}_{n \in \mathbb{N}}$ converges to $f$ in $\mathcal{H}(\kappa)$, where for each $n \in \mathbb{N}$,

$$
p_{n}(z)=\sum_{j=1}^{n} x_{\phi(j)} z^{\phi(j)}, \quad z \in \Omega \text {. }
$$

Let $\epsilon>0$. Since $\left\{E_{\tilde{w}} p_{n}\right\}_{n \in \mathbb{N}^{d}}$ is a Cauchy sequence in $H$, there exists $n_{0} \in \mathbb{N}$ such that $\left\|E_{\tilde{w}} p_{n}-E_{\tilde{w}} p_{n+1}\right\|=\left\|x_{\phi(n+1)}\right\|\left|\tilde{w}^{\phi(n+1)}\right|<\epsilon$ for all $n \geqslant n_{0}$. This proves that $\left\{\tilde{w}^{\alpha} x_{\alpha}\right\}_{\alpha \in \mathbb{N}^{d}}$ is a bounded sequence in $H$. Therefore, we have

$$
\sum_{\alpha \in \mathbb{N}^{d}}\left\|w^{\alpha} x_{\alpha}\right\|=\sum_{\alpha \in \mathbb{N}^{d}}\left\|\tilde{w}^{\alpha} x_{\alpha}\right\| \frac{\left|w^{\alpha}\right|}{\left|\tilde{w}^{\alpha}\right|} \leqslant \sup _{\alpha \in \mathbb{N}^{d}}\left\|\tilde{w}^{\alpha} x_{\alpha}\right\| \sum_{\alpha \in \mathbb{N}^{d}} \frac{\left|w^{\alpha}\right|}{\left|\tilde{w}^{\alpha}\right|}<\infty .
$$

Thus $f$ converges absolutely at $w$ and hence is holomorphic on the interior of $\Omega$. This completes the proof of proposition.

The following theorem shows that the operator-valued multishifts can be realized as the tuple of operators of multiplication by the coordinate functions on a reproducing kernel Hilbert space of vector valued holomorphic functions, provided the set of bounded point evaluations has non-empty interior.

THEOREM 5.7. Let $H$ be a complex separable Hilbert space and $T=\left(T_{1}, \ldots, T_{d}\right)$ be a commuting operator-valued multishift on $\ell_{H}^{2}\left(\mathbb{N}^{d}\right)$ with invertible operator weights $\left\{A_{\alpha}^{(j)}: \alpha \in \mathbb{N}^{d}, j=1, \ldots, d\right\}$. Let $\mathscr{B}=\left\{B_{\alpha} \in \mathcal{G}(H): \alpha \in \mathbb{N}^{d}\right\}$, where $B_{\alpha}$ is as defined in Proposition 2.3. Let $\Omega$ be the set of all bounded point evaluations on $\mathcal{H}^{2}(\mathscr{B})$. Then for every $w \in \Omega$, $\operatorname{ker}\left(\mathscr{M}_{z}^{*}-\bar{w}\right)=\{\kappa(\cdot, w) x: x \in H\}$ and

$$
\bigvee_{w \in \Omega} \operatorname{ker}\left(\mathscr{M}_{z}^{*}-\bar{w}\right)=\mathcal{H}(\kappa)
$$

Moreover, if $\Omega$ has non-empty interior, then $T=\left(T_{1}, \ldots, T_{d}\right)$ is unitarily equivalent to the d-tuple $\mathscr{M}_{z}=\left(\mathscr{M}_{z_{1}}, \ldots, \mathscr{M}_{z_{d}}\right)$ of multiplication operators by the coordinate functions on $\mathcal{H}(\kappa)$.

Proof. Fix $w \in \Omega$. It follows from the general theory of reproducing kernel Hilbert space that for each non-zero $x \in H, \kappa(\cdot, w) x$ is an eigenvector for $\mathscr{M}_{z}^{*}$ corresponding to the eigenvalue $\bar{w}$. Hence, $\{\kappa(\cdot, w) x: x \in H\} \subseteq \operatorname{ker}\left(\mathscr{M}_{z}^{*}-\bar{w}\right)$. Further, suppose that $f \in \operatorname{ker}\left(\mathscr{M}_{z}^{*}-\bar{w}\right) \subseteq \mathcal{H}(\kappa)$. Then $f=\left.g\right|_{\Omega}$ for some $g \in$ $\mathcal{H}^{2}(\mathscr{B})$. For an $H$-valued polynomial $p$ and $j \in\{1, \ldots, d\}$, we get

$$
\begin{aligned}
\left\langle\bar{w}_{j} g, p\right\rangle_{\mathcal{H}^{2}(\mathscr{B})} & =\left\langle\bar{w}_{j} f, p\right\rangle_{\mathcal{H}(\kappa)}=\left\langle\mathscr{M}_{z_{j}}^{*} f, p\right\rangle_{\mathcal{H}(\kappa)}=\left\langle f, z_{j} p\right\rangle_{\mathcal{H}(\kappa)}=\left\langle g, z_{j} p\right\rangle_{\mathcal{H}^{2}(\mathscr{B})} \\
& =\left\langle\mathscr{M}_{z_{j}}^{*} g, p\right\rangle_{\mathcal{H}^{2}(\mathscr{B})} .
\end{aligned}
$$

Thus $g \in \operatorname{ker}\left(\mathscr{M}_{z}^{*}-\bar{w}\right) \subseteq \mathcal{H}^{2}(\mathscr{B})$. It follows from the proof of Theorem 5.1 (ii) that there exists a non-zero vector $x$ in $H$ such that

$$
g(z)=\sum_{\alpha \in \mathbb{N}^{d}} \bar{w}^{\alpha}\left(B_{\alpha}^{*} B_{\alpha}\right)^{-1} x z^{\alpha}
$$

Since $\left.g\right|_{\Omega}=f, f=\kappa(\cdot, w) x$. This shows that $\{\kappa(\cdot, w) x: x \in H\}=\operatorname{ker}\left(\mathscr{M}_{z}^{*}-\bar{w}\right)$. The conclusion in (13) follows from the reproducing property of $\mathcal{H}(\kappa)$. 
For the moreover part, in view of Theorem5.1, it is enough to show that $\mathscr{M}_{z}=$ $\left(\mathscr{M}_{z_{1}}, \ldots, \mathscr{M}_{z_{d}}\right)$ on $\mathcal{H}^{2}(\mathscr{B})$ is unitarily equivalent to $\mathscr{M}_{z}=\left(\mathscr{M}_{z_{1}}, \ldots, \mathscr{M}_{z_{d}}\right)$ on $\mathcal{H}(\kappa)$. To this end, suppose that $\Omega$ has non-empty interior. Define $U: \mathcal{H}^{2}(\mathscr{B}) \rightarrow \mathcal{H}(\kappa)$ by

$$
U f=\left.f\right|_{\Omega}, \quad f \in \mathcal{H}^{2}(\mathscr{B}) .
$$

Since $\Omega$ has non-empty interior, in the view of Proposition 5.6, $U$ is injective. As the inner product in $\mathcal{H}(\kappa)$ is inherited from $\mathcal{H}^{2}(\mathscr{B})$, it follows that $U$ is a surjective isometry. Now for $f \in \mathcal{H}^{2}(\mathscr{B})$ and $j \in\{1, \ldots, d\}$, we have

$$
U \mathscr{M}_{z_{j}} f=U\left(z_{j} f\right)=\left.\left(z_{j} f\right)\right|_{\Omega}=\left.\mathscr{M}_{z_{j}} f\right|_{\Omega}=\mathscr{M}_{z_{j}} U f .
$$

This completes the proof of the theorem.

Let $\Omega$ be a subset of $\mathbb{C}^{d}$ with non-empty interior. Let $\mathcal{H}(\kappa)$ be a reproducing kernel Hilbert space of vector-valued functions on $\Omega$ which are holomorphic on the interior of $\Omega$ with reproducing kernel $\kappa$. Suppose that $\mathscr{M}_{z_{j}}$, the multiplication operator by the coordinate function $z_{j}$, on $\mathcal{H}(\kappa)$ is bounded for all $j=1, \ldots, d$. Then it is easy to see that the $d$-tuple $\mathscr{M}_{z}=\left(\mathscr{M}_{z_{1}}, \ldots, \mathscr{M}_{z_{d}}\right)$ on $\mathcal{H}(\kappa)$ is unitarily equivalent to the $d$-tuple $\mathscr{M}_{z}=\left(\mathscr{M}_{z_{1}}, \ldots, \mathscr{M}_{z_{d}}\right)$ on $\mathcal{H}\left(\left.\kappa\right|_{\Omega_{0}}\right)$ for all non-empty open subset $\Omega_{0}$ of $\Omega$. Let $\Omega_{1}, \Omega_{2} \subseteq \mathbb{C}^{d}$ be such that the interior of $\Omega_{1} \cap \Omega_{2}$ is non-empty. Let $\mathcal{H}\left(\kappa_{1}\right)$ and $\mathcal{H}\left(\kappa_{2}\right)$ be two reproducing kernel Hilbert spaces of vector-valued functions on $\Omega_{1}$ and $\Omega_{2}$ respectively, which are holomorphic on the respective interiors. Thus, in order to characterize the unitary equivalence of $\mathscr{M}_{z}$ on $\mathcal{H}\left(\kappa_{1}\right)$ and $\mathcal{H}\left(\kappa_{2}\right)$, there is no loss of generality if we assume $\Omega_{1}=\Omega_{2}$. In view of this, we have the following theorem.

THEOREM 5.8. Let $H$ be a complex separable Hilbert space and $T=\left(T_{1}, \ldots, T_{d}\right)$, $\tilde{T}=\left(\tilde{T}_{1}, \ldots, \tilde{T}_{d}\right)$ be two commuting operator-valued multishifts on $\ell_{H}^{2}\left(\mathbb{N}^{d}\right)$ with respective invertible operator weights $\left\{A_{\alpha}^{(j)}: \alpha \in \mathbb{N}^{d}, j=1, \ldots, d\right\}$ and $\left\{\tilde{A}_{\alpha}^{(j)}: \alpha \in\right.$ $\left.\mathbb{N}^{d}, j=1, \ldots, d\right\}$. Let $\mathscr{B}=\left\{B_{\alpha} \in \mathcal{G}(H): \alpha \in \mathbb{N}^{d}\right\}$ and $\tilde{\mathscr{B}}=\left\{\tilde{B}_{\alpha} \in \mathcal{G}(H)\right.$ : $\left.\alpha \in \mathbb{N}^{d}\right\}$, where $B_{\alpha}$ and $\tilde{B}_{\alpha}$ are as defined in Proposition 2.3 corresponding to $A_{\alpha}^{(j)}$ and $\tilde{A}_{\alpha}^{(j)}$ respectively. Let $\Omega$ be the set of bounded point evaluations on $\mathcal{H}^{2}(\mathscr{B})$ and $\mathcal{H}^{2}(\tilde{\mathscr{B}})$ with non-empty interior. Then $T$ and $\tilde{T}$ are unitarily equivalent if and only if there exists a unitary operator $U$ on $H$ such that

$$
U B_{\alpha}^{*} B_{\alpha}=\tilde{B}_{\alpha}^{*} \tilde{B}_{\alpha} U \text { for all } \alpha \in \mathbb{N}^{d} .
$$

Proof. It follows from Theorem 5.7 that $T$ is unitarily equivalent to $\mathscr{M}_{z}$ on $\mathcal{H}(\kappa)$ and $\tilde{T}$ is unitarily equivalent to $\mathscr{M}_{z}$ on $\mathcal{H}(\tilde{\kappa})$. Hence, we show that $\mathscr{M}_{z}$ on $\mathcal{H}(\kappa)$ is unitarily equivalent to $\mathscr{M}_{z}$ on $\mathcal{H}(\tilde{\kappa})$ if and only if (14) holds. To this end, suppose that $\mathscr{M}_{z}$ on $\mathcal{H}(\kappa)$ is unitarily equivalent to $\mathscr{M}_{z}$ on $\mathcal{H}(\tilde{\kappa})$. Then there exists a unitary operator $\mathscr{U}: \mathcal{H}(\kappa) \rightarrow \mathcal{H}(\tilde{\kappa})$ such that $\mathscr{U} \mathscr{M}_{z_{j}}=\mathscr{M}_{z_{j}} \mathscr{U}$ for all $j=1, \ldots, d$. Therefore by [11, Theorem 3.7], $\mathscr{U}=\mathscr{M}_{\Phi}$ for some $\Phi: \Omega \rightarrow \mathcal{B}(H)$. Since both $\mathscr{U}$ and $\mathscr{U}^{*}$ intertwine $\mathscr{M}_{z}$, following the arguments of the proof of [9] Theorem 8], we see that $\Phi$ is constant and satisfies $\Phi(z) \kappa(z, w)=\tilde{\kappa}(z, w) \Phi(z), z, w \in \Omega$. Let $\Phi(z)=U$ for all $z \in \Omega$. Then it is easy to see that $U$ is a unitary operator on $H$ and

$$
\tilde{\kappa}(z, w)=U \kappa(z, w) U^{*}, \quad z, w \in \Omega .
$$

Now for $x, y \in H$ and $z, w \in \Omega$, we get

$$
\begin{aligned}
& \left\langle\sum_{\alpha \in \mathbb{N}^{d}}\left(\tilde{B}_{\alpha}^{*} \tilde{B}_{\alpha}\right)^{-1} x z^{\alpha} \bar{w}^{\alpha}, y\right\rangle_{H} \stackrel{12}{=}\langle\tilde{\kappa}(z, w) x, y\rangle_{H}=\left\langle U \kappa(z, w) U^{*} x, y\right\rangle_{H} \\
& =\left\langle\kappa(\cdot, w) U^{*} x, \kappa(\cdot, z) U^{*} y\right\rangle_{\mathcal{H}(\kappa)} \\
& \stackrel{\text { (12) }}{=}\left\langle\sum_{\alpha \in \mathbb{N}^{d}} U\left(B_{\alpha}^{*} B_{\alpha}\right)^{-1} U^{*} x z^{\alpha} \bar{w}^{\alpha}, y\right\rangle_{H} \text {. }
\end{aligned}
$$


Thus (14) holds.

Conversely, suppose that there exists a unitary operator $U$ on $H$ such that (14) holds. Equivalently, as noted above, we have

$$
\tilde{\kappa}(z, w)=U \kappa(z, w) U^{*}, \quad z, w \in \Omega .
$$

Define $\Phi: \Omega \rightarrow \mathcal{B}(H)$ by

$$
\Phi(z)=U, \quad z \in \Omega .
$$

Then from [31, Theorem 6.28], the map $\mathscr{M}_{\Phi}: \mathcal{H}(\kappa) \rightarrow \mathcal{H}(\tilde{\kappa})$ defined by

$$
\left(\mathscr{M}_{\Phi} f\right)(z)=\Phi(z) f(z)=U f(z), \quad f \in \mathcal{H}(\kappa), z \in \Omega .
$$

is bounded. It is easy to see that $\mathscr{M}_{\Phi} \mathscr{M}_{z_{j}}=\mathscr{M}_{z_{j}} \mathscr{M}_{\Phi}$ for all $j=1, \ldots, d$. Using [1. Theorem 3.7], for $z, w \in \Omega$ and $x \in H$, we get

$$
\left(\mathscr{M}_{\Phi} \mathscr{M}_{\Phi}^{*} \tilde{\kappa}(\cdot, w) x\right)(z)=\Phi(z) \kappa(z, w) \Phi(w)^{*} x=U \kappa(z, w) U^{*} x=\tilde{\kappa}(z, w) x .
$$

This shows that $\mathscr{M}_{\Phi}^{*}$ can be extended isometrically on $\mathcal{H}(\tilde{\kappa})$. Since $\Phi(w)=U$ is unitary for all $w \in \Omega$, range of $\mathscr{M}_{\Phi}^{*}$ is

$$
\bigvee_{w \in \Omega}\left\{\kappa(\cdot, w) \Phi(w)^{*} x: x \in H\right\}=\mathcal{H}(\kappa) .
$$

Therefore $\mathscr{M}_{\Phi}$ is unitary. This completes the proof.

Acknowledgment. The authors are grateful to Gadadhar Misra, Sameer Chavan and Soumitra Ghara for several helpful suggestions and constant support. We express our gratitude to the faculty and the administration of Department of Mathematics and Statistics, IIT Kanpur and Department of Mathematics, IISc Bangalore for their warm hospitality during the preparation of this paper. We convey our sincere thanks to Jan Stochel for several useful comments.

\section{References}

[1] W. Arveson, D. Hadwin, T. Hoover and E. Kymala, Circular operators, Indiana Univ. Math. J. 33 (1984), 583-595.

[2] B. Bagchi and G. Misra, The homogeneous shifts, J. Funct. Anal. 204 (2003), 293-319.

[3] A. Bourhim, On the local spectral properties of weighted shift operators, Studia Math. 163 (2004), 41-69.

[4] P. Budzyński, P. Dymek and M. Ptak, Analytic structure of weighted shifts on directed trees, Math. Nachr. 290 (2017), 1612-1629.

[5] S. Chavan, D. Pradhan and S. Trivedi, Multishifts on directed Cartesian product of rooted directed trees, Dissertationes Math. (Rozprawy Mat.) 527 (2017), 1-102.

[6] S. Chavan, D. Pradhan and S. Trivedi, Classification of Drury-Arveson-type Hilbert modules associated with certain directed graphs, J. Operator Theory 81 (2019), 21-60.

[7] S. Chavan and S. Trivedi, An analytic model for left-invertible weighted shifts on directed trees, J. London Math. Soc. 94 (2016), 253-279.

[8] S. Chavan, D. Yakubovich, Spherical tuples of Hilbert space operators, Indiana Univ. Math. J. 64 (2015), 577-612.

[9] L. Chen, On intertwining operators via reproducing kernels, Linear Algebra Appl. 438 (2013), 3661-3666.

[10] R. Curto, Applications of several complex variables to multiparameter spectral theory. Surveys of some recent results in operator theory, Vol. II, 25-90, Pitman Res. Notes Math. Ser. 192, Longman Sci. Tech., Harlow, 1988.

[11] R. Curto and N. Salinas, Generalized Bergman kernels and the Cowen-Douglas theory, Amer. J. Math. 106 (1984), 447-488.

[12] G. Gehér, Asymptotic behaviour and cyclic properties of weighted shifts on directed trees, J. Math. Anal. Appl. 440 (2016), 14-32.

[13] R. Gellar, Shift operators in Banach space, Dissertation, Columbia University, New York, N. Y., 1968.

[14] R. Gellar, Operators commuting with a weighted shift, Proc. Amer. Math. Soc. 23 (1969), 538-545. 
[15] R. Gellar, Cyclic vectors and parts of the spectrum of a weighted shift, Trans. Amer. Math. Soc. 146 (1969), 69-85.

[16] R. Gellar, Circularly symmetric normal and subnormal operators, J. Analyse Math. 32 (1977) 93-117.

[17] S. Grabiner, Weighted shifts and Banach algebras of power series, Amer. J. Math. 97 (1975), 16-42.

[18] R. Gupta, S. Kumar and S. Trivedi, Von Neumann's inequality for commuting operatorvalued multishifts, Proc. American Math. Soc. 147 (2019), 2599-2608.

[19] M. Hartz, Von Neumann's inequality for commuting weighted shifts, Indiana Univ. Math. J. 66 (2017), 1065-1079.

[20] H. Helson, Invariant subspaces of the weighted shift. Hilbert space operators and operator algebras (Proc. Internat. Conf., Tihany, 1970), pp. 271277. Colloq. Math. Soc. Jnos Bolyai, 5, North-Holland, Amsterdam, 1972.

[21] D. Herrero, Eigenvectors and cyclic vectors of bilateral weighted shifts. II. Simply invariant subspaces, Integral Equations Operator Theory 6 (1983), 515-524.

[22] Z. Jabłoński, Hyperexpansive operator valued unilateral weighted shifts, Glasg. Math. J. 46 (2004), 405-416.

[23] Z. Jabłoński, I. Jung and J. Stochel, Weighted shifts on directed trees, Mem. Amer. Math. Soc. 216 (2012), no. 1017, viii+106.

[24] N. P. Jewell and A. R. Lubin, Commuting weighted shifts and analytic function theory in several variables, J. Operator Theory, 1 (1979), 207-223.

[25] A. Lambert, Unitary equivalence and reducibility of invertibly weighted shifts, Bull. Austral. Math. Soc. 5 (1971), 157-173.

[26] A. Lambert, T. Turner, The double commutant of invertibly weighted shifts, Duke Math. J. 39 (1972), 385-389.

[27] W. Majdak and J. Stochel, Weighted shifts on directed semi-trees: an application to creation operators on Segal-Bargmann spaces, Complex Anal. Oper. Theory, 10 (2016), 1427-1452.

[28] T. Miller, V. Miller and M. Neumann, Local spectral properties of weighted shifts, J. Operator Theory 51 (2004), 71-88.

[29] W. Mlak, Note on circular operators. I, II, III, IV, Univ. Iagel. Acta Math. 29 (1992) 145-152, 153-162, 163-170, 171-175.

[30] N. Nikol'skii, Treatise on the shift operator. Spectral function theory. With an appendix by S. V. Hruev [S. V. Khrushchv] and V. V. Peller. Translated from the Russian by Jaak Peetre. Grundlehren der Mathematischen Wissenschaften [Fundamental Principles of Mathematical Sciences], 273. Springer-Verlag, Berlin, 1986. xii+491 pp.

[31] V. Paulsen and M. Raghupathi, An Introduction to the Theory of Reproducing Kernel Hilbert Spaces, Cambridge Studies in Advanced Mathematics, 152. Cambridge University Press, Cambridge, 2016.

[32] A. Shields, Weighted shift operators and analytic function theory, in Topics in Operator Theory, Math. Surveys Monographs, vol. 13, Amer. math. Soc., Providence, RI 1974, 49-128.

Department of Mathematics and Statistics, Indian Institute of Technology KanPUR, INDIA

E-mail address: rajeevg@iitk.ac.in

Department of Mathematics, Indian Institute of Science Bangalore, India

E-mail address: surjitkumar@iisc.ac.in

Department of Mathematics and Statistics, Indian Institute of Technology KanPUR, INDIA

E-mail address: shailtr@iitk.ac.in 